\title{
Supersymmetry on a Euclidean Spacetime Lattice II: Target Theories with Eight Supercharges
}

\author{
Andrew G. Cohen \\ Dept. of Physics, Boston University, 590 Commonwealth Ave, Boston, MA 02215 \\ Email: cohen@andy.bu.edu \\ David B. Kaplan \\ Institute for Nuclear Theory, University of Washington, Seattle, WA 98195-1550 \\ Email: dbkaplan@phys.washington.edu

\section{Emanuel Katz} \\ Dept. of Physics, University of Washington, Seattle, WA 98195-1560 \\ Email: amikatz@phys.washington.edu \\ Mithat Ünsal \\ Institute for Nuclear Theory, University of Washington, Seattle, WA 98195-1550 \\ Email: mithat@phys.washington.edu
}

\begin{abstract}
We formulate Euclidean spacetime lattices whose continuum limits are supersymmetric Yang-Mills theories with eight supercharges in two and three dimensions. The lattice actions are themselves supersymmetric.
\end{abstract}

KEYWORDs: lgf, exs, ft]. 


\section{Contents}

1. Introduction and results 1

$1.1 \mathcal{Q}=8 \mathrm{SYM}$ in $d=2$ dimensions

$1.2 \mathcal{Q}=8 \mathrm{SYM}$ in $d=3$ dimensions

2. The mother theory and the orbifold projection 6

3. The two dimensional lattice 10

3.1 The $\mathcal{Q}=2$ supersymmetry of the $d=2$ lattice

3.2 The $C_{2 v}$ and $U(1)^{4}$ symmetries of the $d=2$ lattice 13

3.3 The continuum limit of the $d=2$ lattice 13

3.4 Renormalization on the $d=2$ lattice 16

3.5 Fixing the moduli 17

4. The three dimensional lattice

4.1 The $d=3$ lattice action and its symmetries 18

4.2 The continuum limit of the $d=3$ lattice 19

4.3 Renormalization on the $d=3$ lattice 21

5. Discussion 22

\section{Introduction and results}

A method for constructing lattice regularizations of certain supersymmetric Yang-Mills (SYM) theories was recently presented in $[1,2]$. The basic idea is to create a lattice which respects a subset of the fermionic symmetries of the SYM target theory; these exact symmetries greatly restrict the form of relevant operators that can be added to the lattice actions, ensuring that the lattice theory flows to the desired target theory in the continuum limit with little or no fine tuning. Realizing this simply stated goal is not so straightforward, however. Naive implementations of fermionic symmetries in lattice models lead to continuum theories which have neither the desired Lorentz symmetry or supersymmetries. Our approach utilizes "orbifold" technology introduced originally in string theory [3], and our lattice constructions borrow most directly from work on the deconstruction of supersymmetric field theories $[4,5]$. As should be expected, the supersymmetric lattices we construct do not look very conventional. Gauge fields appear as noncompact variables, both complex spin zero bosons and Dirac spinors have their components dispersed over both sites and links, and the lattices are not simple cubic 
structures. In many cases we are able to show that attaining the desired continuum limit of such theories involves no fine tuning of operator coefficients. Most of these lattices have the fascinating property that nonabelian chiral symmetries result in the continuum limit without fine tuning, and without having to implement sophisticated fermions, such as domain wall [6] or overlap $[7,8]$. A serious obstacle to the numerical implementation of these lattices exists, however, in that for at least some cases the fermionic determinant is known not to be positive definite [9].

In ref. [1] spatial lattices were constructed for a number of SYM theories with four, eight, and sixteen supercharges, while in ref. [2] the method was applied to the construction of a four supercharge SYM theory on a two dimensional Euclidean spacetime lattice. In this paper we extend this previous work to the study of Euclidean spacetime lattices for SYM theories with eight supercharges in two $[10,11]$ and three dimensions $[12-14]$. We are able to show that the two dimensional example involves no fine tuning, while there are two related operators in the three dimensional case which may receive logarithmic corrections at one loop (but not at higher loops). A companion paper describing lattices for sixteen supercharge SYM theories is in preparation.

For those uninterested in the technical details of our construction of supersymmetric lattices, we begin by simply specifying the target theories we are considering and presenting the corresponding lattice actions, along with the dictionary relating the continuum and lattice variables. We then proceed to describe, first for the two- and then for the three-dimensional case, how we arrive at such lattice actions. We make explicit the exact supersymmetries on the lattice using superfield techniques, and then exploit these symmetries to understand the continuum limit. For additional discussion of the general method, and for citation of prior work on supersymmetric lattice theories, we refer the reader to Refs. [1,2].

\section{1 $\mathcal{Q}=8 \mathrm{SYM}$ in $d=2$ dimensions}

The eight supercharge SYM theories we consider can all be obtained by dimensional reduction of $\mathcal{N}=1 \mathrm{SYM}$ in six dimensions. The two dimensional version, referred to as $(4,4) \mathrm{SYM}$ in $1+1$ Minkowski dimensions, has a continuum action which can be written in Euclidean space in terms of two gauge potentials $v_{1}, v_{2}$, two Dirac fermions $\Psi_{\alpha i}$, where $\alpha=1,2$ is the spinor index and $i=1,2$ is a flavor index, and four real scalars $s_{\mu}=\left\{s_{0}, s_{a}\right\}$ :

$S=\frac{1}{g_{2}^{2}} \int d^{2} x \operatorname{Tr}\left[\frac{1}{4} v_{m n} v_{m n}+\frac{1}{2}\left(D_{m} s_{\mu}\right)^{2}+\bar{\Psi}_{i} \gamma_{m} D_{m} \Psi_{i}+\bar{\Psi}_{i}\left[s_{0}, \Psi_{i}\right]+i \bar{\Psi}_{i} \gamma_{3} \tau_{i j}^{a}\left[s_{a}, \Psi_{j}\right]-\frac{1}{4}\left[s_{\mu}, s_{\nu}\right]^{2}\right]$.

(Throughout this paper, indices $\mu, \nu$ run over $0, \ldots, 3$, indices $a, b, c$ run over $1,2,3$, and indices $i, j, k$ run over values 1,2 ; the letters $m, n$ are reserved for spacetime indices running over the values appropriate in the given context. We will use the letter $\mathfrak{a}$ to refer to the lattice spacing.) All fields in the above expression are $k \times k$ matrices, transforming as adjoints under the $U(k)$ gauge symmetry; $v_{m n}$ is the gauge field strength, $\boldsymbol{\tau}$ are the Pauli matrices acting on 


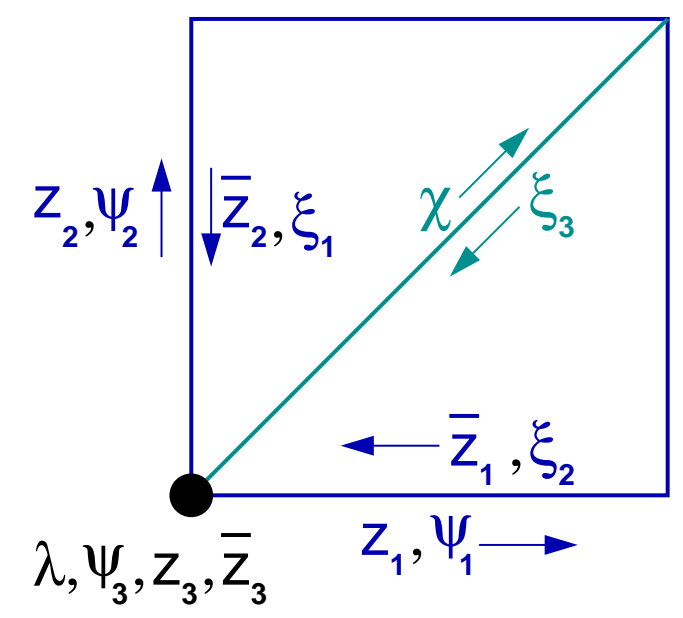

Figure 1: The unit cell of the Euclidean lattice for the two dimensional SYM theory with eight supercharges. The lattice respects two exact supercharges. The figure on the right displays the location of the lattice variables associated with site $\mathbf{n}$ (Latin for complex bosons, Greek for one-component fermions). Arrows signify the orientation of the link variables, or equivalently, the direction of their $\mathbf{r}$ charge vectors, as given in Table 1.

the flavor indices, and the three $\gamma$ matrices satisfy the usual Clifford algebra, $\left\{\gamma_{m}, \gamma_{n}\right\}=2 \delta_{m n}$. This action has an $S U(2)^{3}$ global $R$-symmetry, consisting of the $S U(2) R$-symmetry of $\mathcal{N}=1$ $\mathrm{SYM}$ in six dimensions, and the $S O(4) \sim S U(2) \times S U(2)$ inherited from the $S O(6)$ Lorentz symmetry of the six-dimensional theory after dimensional reduction to two dimensions.

The lattice action we propose for simulating this theory is

$$
\begin{aligned}
& S=\frac{1}{g^{2}} \sum_{\mathbf{n}} \operatorname{Tr}\left[\frac{1}{2}\left(\bar{z}_{i, \mathbf{n}-\hat{\mathbf{e}}_{i}} z_{i, \mathbf{n}-\hat{\mathbf{e}}_{i}}-z_{i, \mathbf{n}} \bar{z}_{i, \mathbf{n}}+\left[\bar{z}_{3, \mathbf{n}}, z_{3, \mathbf{n}}\right]\right)^{2}\right. \\
& +2\left(\left|\epsilon_{i j} z_{i, \mathbf{n}} z_{j, \mathbf{n}+\hat{\mathbf{e}}_{i}}\right|^{2}+\left|z_{i, \mathbf{n}} z_{3, \mathbf{n}+\hat{\mathbf{e}}_{i}}-z_{3, \mathbf{n}} z_{i, \mathbf{n}}\right|^{2}\right) \\
& +\sqrt{2}\left(\Delta_{\mathbf{n}}\left(\lambda, \bar{z}_{a}, \psi_{a}\right)-\Delta_{\mathbf{n}}\left(\chi, \bar{z}_{a}, \xi_{a}\right)+\epsilon_{a b c} \Delta_{\mathbf{n}}\left(\psi_{a}, z_{b}, \xi_{c}\right)\right) \\
& \left.+\frac{\mathfrak{a}^{2} \mu^{2}}{2}\left[\left(z_{i, \mathbf{n}} \bar{z}_{i, \mathbf{n}}-\frac{1}{2 \mathfrak{a}^{2}}\right)^{2}+2 \frac{\bar{z}_{3, \mathbf{n}} z_{3, \mathbf{n}}}{\mathfrak{a}^{2}}\right]\right]
\end{aligned}
$$

where the sum is over sites $\mathbf{n}=\left\{n_{1}, n_{2}\right\}$ with $n_{1,2} \in[1, N]$, with $\hat{\mathbf{e}}_{1}$, $\hat{\mathbf{e}}_{2}$ being unit vectors in $n_{1}$ and $n_{2}$ directions respectively. All variables are $k \times k$ matrices satisfying periodic boundary conditions on the lattice, and there is an independent $U(k)$ symmetry associated with each site, which becomes the $U(k)$ gauge symmetry of the the continuum theory. The indices $i, j$ run over 1 and 2, the subscripts $a, b, c$ take on the values 1,2,3, and all repeated indices are summed. In this expression, the variables $z_{a}$ and $\bar{z}_{a}$ refer to complex bosonic variables and their conjugates, while $\lambda, \chi, \psi_{a}$ and $\xi_{a}$ refer to one-component Grassmann variables. 
The structure of the lattice is shown in Fig. 1. We have defined $\Delta$ as

$$
\Delta_{\mathbf{n}}(A, B, C) \equiv A_{\mathbf{n}}\left(B_{\mathbf{p}} C_{\mathbf{q}}-C_{\mathbf{r}} B_{\mathbf{s}}\right)
$$

where the site variables $\mathbf{p}, \mathbf{q}, \mathbf{r}, \mathbf{s}$ are determined in terms of $\mathbf{n}$ such that each term is gauge invariant, corresponding to a closed path on the lattice of Fig. 1. For example:

$$
\begin{aligned}
\Delta_{\mathbf{n}}\left(\lambda, \bar{z}_{1}, \psi_{1}\right) & =\lambda_{\mathbf{n}}\left(\bar{z}_{1, \mathbf{n}-\hat{\mathbf{e}}_{1}} \psi_{1, \mathbf{n}-\hat{\mathbf{e}}_{1}}-\psi_{1, \mathbf{n}} \bar{z}_{1, \mathbf{n}}\right) \\
\Delta_{\mathbf{n}}\left(\chi, \bar{z}_{3}, \xi_{3}\right) & =\chi_{\mathbf{n}}\left(\bar{z}_{3, \mathbf{n}+\hat{\mathbf{e}}_{1}+\hat{\mathbf{e}}_{2}} \xi_{3, \mathbf{n}}-\xi_{3, \mathbf{n}} \bar{z}_{3, \mathbf{n}}\right) \\
\Delta_{\mathbf{n}}\left(\chi, \bar{z}_{1}, \xi_{1}\right) & =\chi_{\mathbf{n}}\left(\bar{z}_{1, \mathbf{n}+\hat{\mathbf{e}}_{j}} \xi_{1, \mathbf{n}}-\xi_{1, \mathbf{n}+\hat{\mathbf{e}}_{i}} \bar{z}_{1, \mathbf{n}}\right) .
\end{aligned}
$$

The last line in Eq. (1.2) requires special mention. The parameter a appearing in the action has dimension of length; it determines the expectation values of the link variables $\left\langle z_{i}\right\rangle$, which in turn define the lattice spacing. The continuum limit is defined as $\mathfrak{a} \rightarrow 0, N \rightarrow \infty$ while holding fixed the 2-dimensional coupling $g_{2} \equiv g \mathfrak{a}$ and the lattice size $L \equiv N \mathfrak{a}$. Special to two dimensions is that the continuum and thermodynamic limits are not independent, but must satisfy $g_{2} \mathfrak{a} \ln N \rightarrow 0$ (see ref. [2]). The term proportional to $\mu^{2}$ softly breaks the exact supersymmetry, and controls the size of quantum fluctuations of our dynamical lattice spacing, $\delta z_{i} \sim g_{2} /(\mu L)$. To ensure that the fluctuations $\delta z_{i}$ are small compared to their mean value $\left(\delta z_{i} \ll 1 / \mathfrak{a}\right) \mu$ must satisfy the constraint $\mu L \gg g_{2} \mathfrak{a}$ which can be simply satisfied by taking $\mu \sim 1 / L$.

The correspondence between the lattice variables of Eq. (1.2) and the continuum variables in Eq. (1.1) are

$$
\begin{aligned}
& \Psi_{1}=\left(\begin{array}{l}
\xi_{1} \\
\xi_{2}
\end{array}\right), \quad \Psi_{2}=\left(\begin{array}{c}
\lambda \\
-\xi_{3}
\end{array}\right), \quad \bar{\Psi}_{1}=\left(\begin{array}{ll}
-\chi \psi_{3}
\end{array}\right), \quad \bar{\Psi}_{2}=\left(\begin{array}{ll}
\psi_{1} & \psi_{2}
\end{array}\right) . \\
& \left(\begin{array}{l}
s_{0} \\
s_{1} \\
s_{2} \\
s_{3}
\end{array}\right)=\left(\begin{array}{c}
\phi_{1} \\
-\left(z_{3}+\bar{z}_{3}\right) / \sqrt{2} \\
i\left(z_{3}-\bar{z}_{3}\right) / \sqrt{2} \\
\phi_{2}
\end{array}\right), \quad \phi_{i} \equiv \sqrt{2} \operatorname{Re}\left(z_{i}-\frac{1}{\sqrt{2} \mathfrak{a}}\right), \quad v_{m}=\operatorname{Im}\left[z_{m}\right] .
\end{aligned}
$$

\section{$1.2 \mathcal{Q}=8 \mathrm{SYM}$ in $d=3$ dimensions}

The target theory in three dimensions consists of a gauge potential $v_{m}$, where now $m=$ $0,1,2$; two Dirac fermions $\Psi_{\alpha i}$; and three real scalars $\phi_{a}, a=1,2,3$. The action respects an $S U(2) \times S U(2)$ global symmetry under which $v_{m}$ is a $(1,1), \Psi$ and $\bar{\Psi}$ together form a $(2,2)$, and $\phi_{a}=(3,1)$. The action is

$$
S=\frac{1}{g_{3}^{2}} \int d^{3} x \operatorname{Tr}\left[\frac{1}{4} v_{m n} v_{m n}+\frac{1}{2}\left(D_{m} \phi_{a}\right)^{2}+\bar{\Psi}_{i} \sigma_{m} D_{m} \Psi_{i}-\bar{\Psi}_{i} \tau_{i j}^{a} \cdot\left[\phi_{a}, \Psi_{j}\right]-\frac{1}{4}\left[\phi_{a}, \phi_{b}\right]^{2}\right]
$$




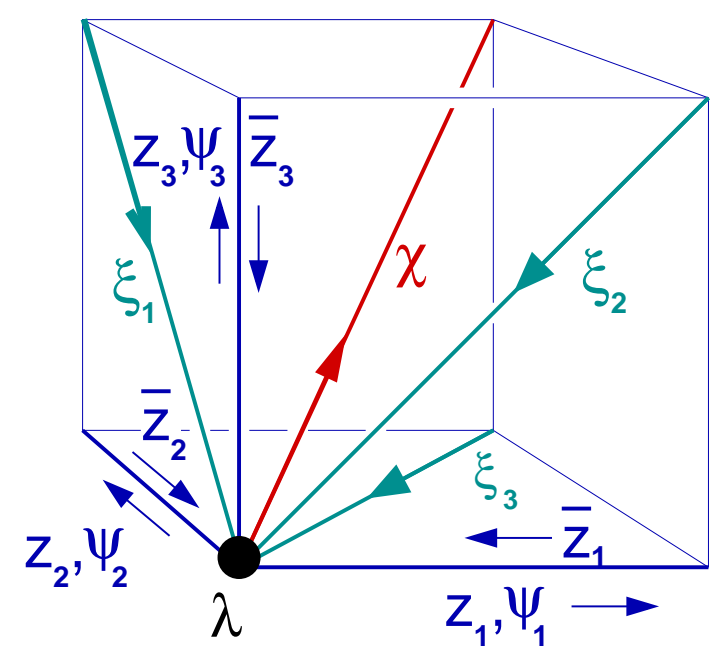

Figure 2: The unit cell of the Euclidean lattice for the three dimensional SYM theory with eight supercharges. Latin and Greek letters refer to complex boson and one-component Grassmann variables respectively. The lattice respects one exact supercharge.

The unit cell for our lattice version of this theory is shown in Fig. 2. The action is given by

$$
\begin{aligned}
S= & \frac{1}{g^{2}} \sum_{\mathbf{n}} \operatorname{Tr}\left[\frac{1}{2}\left(\bar{z}_{a, \mathbf{n}-\hat{\mathbf{e}}_{a}} z_{a, \mathbf{n}-\hat{\mathbf{e}}_{a}}-z_{a, \mathbf{n}} \bar{z}_{a, \mathbf{n}}\right)^{2}+2\left|\epsilon_{a b c} z_{a, \mathbf{n}} z_{b, \mathbf{n}+\hat{\mathbf{e}}_{a}}\right|^{2}\right. \\
& \left.+\sqrt{2}\left(\Delta_{\mathbf{n}}\left(\lambda, \bar{z}_{a}, \psi_{a}\right)-\Delta_{\mathbf{n}}\left(\chi, \bar{z}_{a}, \xi_{a}\right)+\epsilon_{a b c} \Delta_{\mathbf{n}}\left(\psi_{a}, z_{b}, \xi_{c}\right)\right)+\mathfrak{a}^{2} \mu^{2}\left(z_{b, \mathbf{n}} \bar{z}_{b, \mathbf{n}}-\frac{1}{2 \mathfrak{a}^{2}}\right)^{2}\right]
\end{aligned}
$$

The operator $\Delta$ is defined as in Eq. (1.3); however, terms differ from the two dimensional case due to the different lattice structure. Now, for example

$$
\Delta_{\mathbf{n}}\left(\chi, \bar{z}_{3}, \xi_{3}\right)=\chi_{\mathbf{n}}\left(\bar{z}_{3, \mathbf{n}+\hat{\mathbf{e}}_{1}+\hat{\mathbf{e}}_{2}} \xi_{3, \mathbf{n}}-\xi_{3, \mathbf{n}+\hat{\mathbf{e}}_{3}} \bar{z}_{3, \mathbf{n}}\right)
$$

corresponding to the signed sum of the two triangular plaquettes on either side for the $\chi$ link in the $\chi-\xi_{3}-\bar{z}_{3}$ plane in Fig. 2. The lattice action, with the exception of the soft SUSY breaking terms proportional to $\mu^{2}$ which fix the lattice size, respects a single supercharge. In the case of this three dimensional lattice, the continuum limit is defined by $\mathfrak{a} \rightarrow 0, g \rightarrow \infty$, $\mathfrak{a}^{3} g^{2} \rightarrow g_{3}^{2}, N \rightarrow \infty$, keeping $g_{3}^{2}$ and $L=\mathfrak{a} N$ fixed. The infinite volume limit involves taking $L \rightarrow \infty$ and $\mu \rightarrow 0$, with $\mu L$ held fixed and satisfying $\mu L \gg\left(g_{3} \mathfrak{a} / \sqrt{L}\right)$; as in the two dimensional case, one can simply fix $\mu L \sim 1$.

In terms of the lattice fields in Eq. (1.8), the continuum fields in Eq. (1.7) are given by

$$
v_{m}=\sqrt{2} \operatorname{Im}\left[z_{m}\right], \quad \phi_{a}=\sqrt{2} \operatorname{Re}\left[z_{a}-\frac{1}{\sqrt{2} \mathfrak{a}}\right]
$$


for the bosons, and for the fermions

$$
\begin{aligned}
& \Psi_{1}=\frac{1}{\sqrt{2}}\left(\begin{array}{c}
\psi_{3}-i \chi \\
\psi_{1}+i \psi_{2}
\end{array}\right), \quad \bar{\Psi}_{1}=\frac{i}{\sqrt{2}}\left(\begin{array}{c}
\xi_{3}+i \lambda \\
\xi_{1}-i \xi_{2}
\end{array}\right)^{T} \\
& \Psi_{2}=\frac{1}{\sqrt{2}}\left(\begin{array}{c}
-\psi_{1}+i \psi_{2} \\
\psi_{3}+i \chi
\end{array}\right), \quad \bar{\Psi}_{2}=\frac{i}{\sqrt{2}}\left(\begin{array}{c}
-\xi_{1}-i \xi_{2} \\
\xi_{3}-i \lambda
\end{array}\right)^{T}
\end{aligned}
$$

in a basis where the three gamma matrices are just the Pauli matrices,

$$
\gamma_{m}=\sigma_{m}
$$

In the following sections we derive these results and analyze the renormalization properties of our lattices.

\section{The mother theory and the orbifold projection}

The lattices for $\mathcal{Q}=8 \mathrm{SYM}$ theories in two and three dimensions arise from the $\mathcal{Q}=8$ mother theory obtained by dimensionally reducing $\mathcal{N}=1 \mathrm{SYM}$ from six (Euclidean) dimensions (a theory consisting of a six component gauge potential and a four component Weyl adjoint fermion) to zero dimensions. The action for the mother theory may be written as

$$
S=\frac{1}{g^{2}}\left(\frac{1}{4} \operatorname{Tr} v_{m n} v_{m n}+\operatorname{Tr} \bar{\psi} \bar{\Sigma}_{m}\left[v_{m}, \psi\right]\right)
$$

where $m, n=0, \ldots, 5, \psi$ and $\bar{\psi}$ are independent complex four-component spinors and $v_{m n}=$ $i\left[v_{m}, v_{n}\right]$. The variables $\psi, \bar{\psi}$ and $v_{m}$ are all matrices transforming as adjoints under a Lie group $\mathcal{G}$. The six $\bar{\Sigma}_{m}$ matrices are defined as

$$
\bar{\Sigma}_{m}=\{1, i \gamma\}=\Sigma_{m}^{\dagger}
$$

where $\gamma$ are the five four-dimensional gamma matrices obeying the Clifford algebra for $S O(5)$.

The global symmetry of the action Eq. (2.1) is $G_{R}=S O(6) \times S U(2)$. The $S O(6)$ symmetry is just the (Euclidean version of the) inherited six dimensional Lorentz symmetry, but the form of the action Eq. (2.1) makes the $S U(2)$ symmetry far from manifest. We therefore define $C$ to be the charge conjugation matrix for $S O(5)$ satisfying

$$
C=C^{\dagger}=C^{-1}=-C^{T}, \quad C \gamma C=+\gamma^{T},
$$

and define the $4 \times 2$ Grassmann field

$$
\Psi=\left(\begin{array}{ll}
\psi & C \bar{\psi}^{T}
\end{array}\right)
$$


In the above expression, the transpose in $\bar{\psi}^{T}$ affects the Dirac indices only, and not the gauge indices. Then in terms of $\Psi$, the action Eq. (2.1) may be reexpressed as

$$
\frac{1}{g^{2}}\left(\frac{1}{4} \operatorname{Tr} v_{m n} v_{m n}-\frac{i}{2} \operatorname{Tr} \sigma_{2} \Psi^{T} C \bar{\Sigma}_{m}\left[v_{m}, \Psi\right]\right)
$$

It is now evident that this mother theory has a global $G_{R}=S O(6) \times S U(2)$ symmetry under which

$$
v \rightarrow \Omega v \Omega^{-1}, \quad \Psi \rightarrow \Omega \Psi U^{\dagger}, \quad \text { with } \quad v \equiv C \bar{\Sigma}_{m} v_{m}
$$

where $\Omega \in S O(6)$ and $U \in S U(2)$.

The eight supersymmetry transformations may be parametrized by a constant Grassmann $4 \times 2$ dimensional spinor $\kappa$. The action Eq. (2.5) is invariant under the supersymmetry transformations

$$
\delta v_{m}=\operatorname{Tr}_{2} \sigma_{2} \kappa^{T} C \bar{\Sigma}_{m} \Psi, \quad \delta \Psi=-i v_{m n} \Sigma_{m n} \kappa, \quad \delta \Psi^{T}=-i v_{m n} \kappa^{T} \Sigma_{m n}^{T},
$$

where $\operatorname{Tr}_{2}$ means a trace over the $2 \times 2$ matrix, and not over gauge indices, and we have defined the matrices

$$
\Sigma_{m n}=\frac{i}{4}\left(\Sigma_{m} \bar{\Sigma}_{n}-\Sigma_{n} \bar{\Sigma}_{m}\right)
$$

A lattice may be created out of the matrices of the mother theory, following the procedure in [2]. To create a $d$-dimensional lattice with $N^{d}$ sites and possessing a $U(k)$ gauge symmetry, we take the group $\mathcal{G}$ of the mother theory to be $U\left(k N^{d}\right)$. The variables of the mother theory are then all $k N^{d} \times k N^{d}$ matrices. A given matrix variable $\Phi$ of the mother theory is most conveniently labeled not with two indices, but as $\Phi_{i j}^{(\boldsymbol{\mu}, \boldsymbol{\nu})}$, where $i, j$ run from $1, \ldots, k$, while $\boldsymbol{\mu}$ and $\boldsymbol{\nu}$ are $d$-component vectors, with each element running over $1, \ldots, N$. We then define a particular $\Gamma=Z_{N}^{d}$ subgroup of the $G_{R} \times \mathcal{G}$ symmetry of the mother theory, whose generators $\hat{\gamma}_{a}$ act on the field $\Phi$ as

$$
\hat{\gamma}_{a} \Phi=e^{2 \pi i r_{a} / N} \mathcal{C}^{(a)} \Phi \mathcal{C}^{(a)^{-1}} \quad \text { with } \quad a=1, \ldots, d .
$$

In this expression, $e^{2 \pi i r_{a} / N} \in G_{R}$ and $\mathcal{C}^{(a)} \in \mathcal{G}$. The $\mathcal{C}^{(a)}$ are referred to as "clock" matrices. They can be written compactly as the direct product of $d$ rank- $N$ matrices and one rank- $k$ matrix:

$$
\begin{aligned}
& \mathcal{C}^{(1)}=\Omega \otimes \mathbf{1}_{N} \otimes \cdots \otimes \mathbf{1}_{N} \otimes \mathbf{1}_{k}, \\
& \mathcal{C}^{(2)}=\mathbf{1}_{N} \otimes \Omega \otimes \cdots \otimes \mathbf{1}_{N} \otimes \mathbf{1}_{k}, \\
& \cdots \\
& \mathcal{C}^{(d)}=\mathbf{1}_{N} \otimes \mathbf{1}_{N} \otimes \cdots \otimes \Omega \otimes \mathbf{1}_{k},
\end{aligned}
$$

where $\mathbf{1}_{m}$ signifies a rank- $m$ unit matrix, and $\Omega$ is the rank- $N$ unitary matrix

$$
\Omega=\left(\begin{array}{cccc}
\omega & & & \\
& \omega^{2} & & \\
& & \ddots & \\
& & & \omega^{N}
\end{array}\right) \quad \text { with } \quad \omega \equiv e^{2 \pi i / N}
$$


When multiplied on the left of $\Phi_{i j}^{(\boldsymbol{\mu}, \boldsymbol{\nu})} \mathcal{C}^{(m)}$ acts nontrivially only on the $m^{\text {th }}$ component of the index vector $\boldsymbol{\mu}$, while $\mathcal{C}^{(m)^{-1}}$ acts nontrivially only on the $m^{\text {th }}$ component of the index vector $\boldsymbol{\nu}$ when multiplied on the right.

The integer charges $\mathbf{r}=\left\{r_{1}, \ldots, r_{d}\right\}$ in Eq. (2.9) are constructed from the Cartan subalgebra of $G_{R}=S O(6) \times S U(2)$. Our specific choice of these charges is discussed below. We will choose a basis for the variables of the mother theory such that each $\Phi_{i j}^{(\boldsymbol{\mu}, \boldsymbol{\nu})}$ is an eigenstate of these $d$ charges, and so each factor $e^{2 \pi i r_{a} / N}$ in Eq. (2.9) will be a simple phase, and will act trivially on the indices of $\Phi$

The orbifold projection eliminates the components of the mother theory fields which are not invariant under the discrete $\Gamma$ transformation defined in Eq. (2.9). The projection operator may be written as

$$
\hat{P}=\frac{1}{N^{d}} \sum_{m_{1}=1}^{N} \cdots \sum_{m_{d}=1}^{N}\left(\hat{\gamma}_{1}\right)^{m_{1}} \cdots\left(\hat{\gamma}_{d}\right)^{m_{d}} .
$$

The "daughter" theory is obtained by replacing every field $\Phi$ in the action of the mother theory by its projection $\tilde{\Phi}=\hat{P} \Phi$. Each projected field $\tilde{\Phi}$ is very sparse, consisting only of $N^{d}$ nonzero (unconstrained) $k \times k$ blocks; the position of these nonzero blocks within the original rank $k N^{d}$ matrix is determined by the $\mathbf{r}$ charges of $\Phi$. In particular, the nonzero blocks in $\tilde{\Phi}_{i j}^{(\boldsymbol{\mu}, \boldsymbol{\nu})}$ occur only for

$$
\nu=\mu+\mathbf{r}
$$

Recall that $\boldsymbol{\mu}, \boldsymbol{\nu}$ and $\mathbf{r}$ are each $d$-component vectors with integer components running from 1 to $N$. We can therefore consider these nonzero $k \times k$ blocks as lattice variables of a $d-$ dimensional, $N^{d}$-site lattice. We label each lattice site by a vector $\boldsymbol{\mu}$. Each nontrivial block in $\tilde{\Phi}$ is then a variable residing on the link between sites $\boldsymbol{\mu}$ and $\boldsymbol{\mu}+\mathbf{r}$. Since the orbifold projection breaks the symmetry $\mathcal{G}$ of the mother theory down to an independent $U(k)$ symmetry for each of the $N^{d}$ sites of the lattice, a link variable between sites $\boldsymbol{\mu}$ and $\boldsymbol{\mu}+\mathbf{r}$ transforms under the bilinear $(\square, \bar{\square})$ representation of the $U(k) \times U(k)$ symmetry associated with sites $\boldsymbol{\mu}$ and $\boldsymbol{\mu}+\mathbf{r}$. A variable of the mother theory with $\mathbf{r}=0$ corresponds to site variables on the lattice, each one transforming under the adjoint representation of the $U(k)$ symmetry associated with that site.

In order to make this explicit, we must choose the $\mathbf{r}$ charges, and express the variables of the mother theory in terms of eigenstates of these charges. We begin by defining a basis $q_{1}, \ldots, q_{4}$ for the Cartan sub-algebra of the rank 4 group $G_{R}=S O(6) \times S U(2)$. The generators of the $S O(6)$ are just the matrices $\Sigma_{m n}$ defined in Eq. (2.8), while we denote the three generators of the $S U(2)$ as $\frac{1}{2} \boldsymbol{\tau}$. We choose as our basis the four mutually commuting charges

$$
q_{1}=\Sigma_{01}, \quad q_{2}=\Sigma_{23}, \quad q_{3}=\Sigma_{45}, \quad q_{4}=\frac{1}{2} \tau_{3} .
$$

Next we define the three bosonic variables $z_{a}$ and their Hermitean conjugates $\bar{z}_{a}$, which are linear combinations of the six gauge fields $v_{m}$ of the mother theory, and which are eigenstates 
of the $q_{i}$ :

$$
\begin{aligned}
& z_{1}=+i \frac{v_{0}+i v_{1}}{\sqrt{2}}, \quad z_{2}=\frac{v_{2}+i v_{3}}{\sqrt{2}}, \quad z_{3}=\frac{v_{4}+i v_{5}}{\sqrt{2}}, \\
& \bar{z}_{1}=-i \frac{v_{0}-i v_{1}}{\sqrt{2}}, \quad \bar{z}_{2}=\frac{v_{2}-i v_{3}}{\sqrt{2}}, \quad \bar{z}_{3}=\frac{v_{4}-i v_{5}}{\sqrt{2}} .
\end{aligned}
$$

The $q_{i}$ charges of these variables are given in Table 1.

At this point it is convenient to use the particular basis for the $S O(5)$ gamma matrices of Eq. (2.2)

$$
\begin{aligned}
& \gamma_{1}=-\sigma_{3} \otimes 1, \quad \gamma_{2}=\sigma_{1} \otimes \sigma_{1}, \quad \gamma_{3}=-\sigma_{1} \otimes \sigma_{2}, \\
& \gamma_{4}=-\sigma_{1} \otimes \sigma_{3}, \quad \gamma_{5}=\sigma_{2} \otimes 1, \quad C=\sigma_{3} \otimes \sigma_{2} .
\end{aligned}
$$

In this basis the $q_{i}$ are diagonal,

$$
q_{1}=\frac{1}{2} \sigma_{3} \otimes 1, \quad q_{2}=\frac{1}{2} 1 \otimes \sigma_{3}, \quad q_{3}=\frac{1}{2} \sigma_{3} \otimes \sigma_{3}, \quad q_{4}=\frac{1}{2} \tau_{3},
$$

and the gauge potential matrix takes the particularly simple form

$$
v=C \bar{\Sigma}_{m} v_{m}=\sqrt{2}\left(\begin{array}{cccc}
0 & \bar{z}_{1} & \bar{z}_{2} & \bar{z}_{3} \\
-\bar{z}_{1} & 0 & z_{3} & -z_{2} \\
-\bar{z}_{2} & -z_{3} & 0 & z_{1} \\
-\bar{z}_{3} & z_{2} & -z_{1} & 0
\end{array}\right) .
$$

Furthermore, the individual components of $\Psi$ are $q_{i}$ eigenstates in this basis. We label them as

$$
\Psi=\left(\begin{array}{cc}
\lambda & \chi \\
\xi_{1} & \psi_{1} \\
\xi_{2} & \psi_{2} \\
\xi_{3} & \psi_{3}
\end{array}\right)
$$

and give their $q_{i}$ charges in Table 1 as well. In terms of these variables, the action of the mother theory Eq. (2.1) becomes

$$
S=\frac{1}{g^{2}} \operatorname{Tr}\left[\frac{1}{2}\left[\bar{z}_{a}, z_{a}\right]^{2}+2\left|\epsilon_{a b c} z_{a} z_{b}\right|^{2}+\sqrt{2}\left(\lambda\left[\bar{z}_{a}, \psi_{a}\right]-\chi\left[\bar{z}_{a}, \xi_{a}\right]+\epsilon_{a b c} \psi_{a}\left[z_{b}, \xi_{c}\right]\right)\right] .
$$

The next task is to construct the $\mathbf{r}$ charges out of independent linear combinations of the $q_{i}$. The criterion for choosing one combination over the other is that (i) all $r_{a}$ charges must be integer for Eq. (2.9) to define a $Z_{N}$ transformation; (ii) all $r_{a}$ charges should be 0 or \pm 1 if we only want interactions between neighboring sites of the lattice; (iii) as explained in Refs. [1,2], the number of unbroken supersymmetries equals the number of $\mathbf{r}=0$ Grassmann variables, so we want the maximum number of fermion components (half of them) to have $r_{a}=0$ for each $a$. These considerations lead us to define the three $r_{a}$ charges:

$$
r_{1}=q_{1}+q_{4}, \quad r_{2}=q_{2}+q_{4}, \quad r_{3}=q_{3}+q_{4} .
$$

Table 1 lists the $\mathbf{r}$ charges for each of the variables in the mother theory. 


\begin{tabular}{|r||r|r|r||r|r|r|r||r|r|r|r|}
\hline & $z_{1}$ & $z_{2}$ & $z_{3}$ & $\lambda$ & $\xi_{1}$ & $\xi_{2}$ & $\xi_{3}$ & $\chi$ & $\psi_{1}$ & $\psi_{2}$ & $\psi_{3}$ \\
\hline$q_{1}$ & +1 & 0 & 0 & $+\frac{1}{2}$ & $+\frac{1}{2}$ & $-\frac{1}{2}$ & $-\frac{1}{2}$ & $+\frac{1}{2}$ & $+\frac{1}{2}$ & $-\frac{1}{2}$ & $-\frac{1}{2}$ \\
$q_{2}$ & 0 & +1 & 0 & $+\frac{1}{2}$ & $-\frac{1}{2}$ & $+\frac{1}{2}$ & $-\frac{1}{2}$ & $+\frac{1}{2}$ & $-\frac{1}{2}$ & $+\frac{1}{2}$ & $-\frac{1}{2}$ \\
$q_{3}$ & 0 & 0 & +1 & $+\frac{1}{2}$ & $-\frac{1}{2}$ & $-\frac{1}{2}$ & $+\frac{1}{2}$ & $+\frac{1}{2}$ & $-\frac{1}{2}$ & $-\frac{1}{2}$ & $+\frac{1}{2}$ \\
$q_{4}$ & 0 & 0 & 0 & $-\frac{1}{2}$ & $-\frac{1}{2}$ & $-\frac{1}{2}$ & $-\frac{1}{2}$ & $+\frac{1}{2}$ & $+\frac{1}{2}$ & $+\frac{1}{2}$ & $+\frac{1}{2}$ \\
\hline$r_{1}$ & +1 & 0 & 0 & 0 & 0 & -1 & -1 & +1 & +1 & 0 & 0 \\
$r_{2}$ & 0 & +1 & 0 & 0 & -1 & 0 & -1 & +1 & 0 & +1 & 0 \\
$r_{3}$ & 0 & 0 & +1 & 0 & -1 & -1 & 0 & +1 & 0 & 0 & +1 \\
\hline
\end{tabular}

Table 1: The $q_{1, \ldots, 4}$ charges of the boson, fermion, and auxiliary fields of the $\mathcal{Q}=8$ mother theory under the $U(1)^{4}$ subgroup of $G_{R}=S O(6) \times S U(2)$. The $r_{1,2,3}$ charges are the linear combinations of the $q$ charges which define the possible orbifold projections. The $\bar{z}_{a}$ fields have charges opposite to those of their unbarred counterparts.

By choosing the orbifold group $\Gamma$ to be $Z_{N}, Z_{N}^{2}$ or $Z_{N}^{3}$ we will obtain one-, two-, or threedimensional lattices, possessing four, two or one supersymmetries respectively. We could in principle create a four dimensional lattice, but it would not respect any exact supersymmetry. In the remainder of this article we focus on the two- and three-dimensional lattices.

\section{The two dimensional lattice}

To create a two dimensional lattice from the $\mathcal{Q}=8$ mother theory, which will describe the $(4,4)$ SYM theory in the two-dimensional continuum, we orbifold by $Z_{N} \times Z_{N}$, where the two $Z_{N}$ transformations are determined by the charges $r_{1}$ and $r_{2}$ in Table 1. The lattice we obtain takes the form shown in Figure 1, with $\left\{z_{3}, \bar{z}_{3}, \lambda\right\}$ residing at the sites; $\left\{z_{1}, \bar{z}_{1}, \xi_{2}, \psi_{1}\right\}$ on the $\hat{\mathbf{e}}_{1}$-links, $\left\{z_{2}, \bar{z}_{2}, \xi_{1}, \psi_{2}\right\}$ on the $\hat{\mathbf{e}}_{2}$-links; and $\left\{\xi_{3}, \psi_{3}\right\}$ along the diagonal links. The lattice action in terms of the component fields follows immediately from our $\mathbf{r}$ charge assignments and Eq. (2.20), and is the action of Eq. (1.2), with the omission of the soft supersymmetry breaking term proportional to $\mu$. The symmetries of this action include $\mathcal{Q}=2$ supersymmetry, the $U(k)$ gauge symmetry, a $U(1)^{4}$ global symmetry (generated by the four $q_{i}$ charges), and a $C_{2 v}$ lattice symmetry. The generators of the $C_{2 v}$ symmetry consist of reflections about the diagonal axis, $\sigma_{d}$, and $\pi$ rotations about the normal to the lattice, $C_{2}$. It is worthwhile to make these symmetries manifest so that we can more easily analyze the approach to the continuum.

\subsection{The $\mathcal{Q}=2$ supersymmetry of the $d=2$ lattice}

We begin by rewriting the action in a superfield formalism, which will make the $\mathcal{Q}=2$ symmetry manifest. 
To find the exact supersymmetry transformations, we need only find the subset of the full supersymmetry transformations in Eq. (2.7) which commute with the $\mathbf{r}=\left\{r_{1}, r_{2}\right\}$ charges. One finds these to correspond to restricting the supersymmetric parameter $\kappa$ in Eq. (2.7) to the form

$$
\kappa=\left(\begin{array}{cc}
\eta & 0 \\
0 & 0 \\
0 & 0 \\
0 & \bar{\eta}
\end{array}\right)
$$

where $\eta$ and $\bar{\eta}$ are independent one-component Grassman parameters. Then the supersymmetry transformations in the mother theory in Eq. (2.7), restricted to $\kappa$ as given above, are

$$
\begin{aligned}
\delta z_{i} & =i \sqrt{2} \eta \psi_{i} & \delta \bar{z}_{i} & =i \epsilon_{i j} \sqrt{2} \bar{\eta} \xi_{j} \\
\delta \psi_{i} & =2 i \bar{\eta}\left[z_{i}, \bar{z}_{3}\right] & \delta \xi_{i} & =-2 i \epsilon_{i j} \eta\left[\bar{z}_{j}, \bar{z}_{3}\right] \\
\delta z_{3} & =i \sqrt{2}\left(\eta \psi_{3}+\bar{\eta} \lambda\right) & \delta \bar{z}_{3} & =0 \\
\delta \psi_{3} & =i \bar{\eta}\left(\left[\bar{z}_{i}, z_{i}\right]-\left[\bar{z}_{3}, z_{3}\right]\right) & \delta \lambda & =-i \eta\left(\left[\bar{z}_{i}, z_{i}\right]+\left[\bar{z}_{3}, z_{3}\right]\right) \\
\delta \chi & =2 i \bar{\eta}\left[z_{1}, z_{2}\right] & \delta \xi_{3} & =-2 i \eta\left[\bar{z}_{1}, \bar{z}_{2}\right] .
\end{aligned}
$$

The fact that both sides of these transformations carry the same $\mathbf{r}$ charges is equivalent to the statement that the orbifold projection leaves these supersymmetry transformations unbroken. We have grouped together fields according to their $\mathbf{r}$ charges: the three groups above reside on the lattice at the $x$ - and $y$-links, sites, and diagonal links respectively.

The supersymmetry transformations Eq. (3.2) may be written in terms of two supercharges $Q$ and $\bar{Q}$ as

$$
\delta=i \eta Q+i \bar{\eta} \bar{Q}
$$

The $Q$ and $\bar{Q}$ supercharges may be realized in terms of the independent Grassmann coordinates $\theta$ and $\bar{\theta}$ by defining

$$
Q=\frac{\partial}{\partial \theta}+\sqrt{2} \bar{\theta}\left[\bar{z}_{3}, \cdot\right], \quad \bar{Q}=\frac{\partial}{\partial \bar{\theta}}+\sqrt{2} \theta\left[\bar{z}_{3}, \cdot\right]
$$

This requires the introduction of three auxiliary fields $d, G$ and $\bar{G}$, since the last two lines of Eq. (3.2) are only compatible with $Q^{2}=\bar{Q}^{2}=0$ after invoking the equations of motion. The auxiliary fields modify the variations of $\lambda, \chi, \psi_{3}$, and $\xi_{3}$ which now read:

$$
\begin{aligned}
\delta \psi_{3} & =i \bar{\eta}\left(\left[\bar{z}_{i}, z_{i}\right]-\left[\bar{z}_{3}, z_{3}\right]-i d\right) & \delta \lambda & =-i \eta\left(\left[\bar{z}_{i}, z_{i}\right]+\left[\bar{z}_{3}, z_{3}\right]+i d\right) \\
\delta \chi & =i \bar{\eta}\left(2\left[z_{1}, z_{2}\right]-\sqrt{2} \bar{G}\right) & \delta \xi_{3} & =-i \eta\left(2\left[\bar{z}_{1}, \bar{z}_{2}\right]-\sqrt{2} G\right)
\end{aligned}
$$

while the auxiliary fields vary in such a way as to ensure $Q^{2}=\bar{Q}^{2}=0$ :

$$
\begin{aligned}
\delta \bar{G} & =2 i \eta \epsilon_{i j}\left[z_{i}, \psi_{j}\right] \\
\delta G & =-2 i \bar{\eta}\left[z_{i}, \xi_{i}\right] \\
\delta d & =-\sqrt{2} \eta\left(\left[\bar{z}_{i}, \psi_{i}\right]+\left[\bar{z}_{3}, \psi_{3}\right]\right)-i \sqrt{2} \bar{\eta}\left(\epsilon_{i j}\left[z_{i}, \xi_{j}\right]+\left[\bar{z}_{3}, \lambda\right]\right) .
\end{aligned}
$$


We can also define supersymmetric derivatives which anticommute with the $Q$ 's:

$$
\mathcal{D}=\frac{\partial}{\partial \theta}-\sqrt{2} \bar{\theta}\left[\bar{z}_{3}, \cdot\right], \quad \overline{\mathcal{D}}=\frac{\partial}{\partial \bar{\theta}}-\sqrt{2} \theta\left[\bar{z}_{3}, \cdot\right]
$$

All of the link fields may then be combined into chiral superfields:

$$
\begin{aligned}
\mathbf{Z}_{i} & =z_{i}+\sqrt{2} \theta \psi_{i}-\sqrt{2} \theta \bar{\theta}\left[\bar{z}_{3}, z_{i}\right], & \overline{\mathbf{Z}}_{i} & =\bar{z}_{i}+\sqrt{2} \bar{\theta} \epsilon_{i j} \xi_{j}+\sqrt{2} \theta \bar{\theta}\left[\bar{z}_{3}, \bar{z}_{i}\right], \\
\mathbf{\Xi} & =\xi_{3}+\sqrt{2} \theta\left(G-\sqrt{2}\left[\bar{z}_{1}, \bar{z}_{2}\right]\right)-\sqrt{2} \theta \bar{\theta}\left[\bar{z}_{3}, \xi_{3}\right], & \bar{\Xi} & =\chi-\sqrt{2} \bar{\theta}\left(\bar{G}-\sqrt{2}\left[z_{1}, z_{2}\right]\right)+\sqrt{2} \theta \bar{\theta}\left[\bar{z}_{3}, \chi\right] .
\end{aligned}
$$

satisfying the chiral constraints $\overline{\mathcal{D}} \mathbf{Z}_{i}=\overline{\mathcal{D}} \boldsymbol{\Xi}=0$, and $\mathcal{D} \overline{\mathbf{Z}}_{i}=\mathcal{D} \overline{\boldsymbol{\Xi}}=0$. The site variables reside in a non-chiral superfield

$$
\mathbf{S}=z_{3}+\sqrt{2} \theta \psi_{3}+\sqrt{2} \bar{\theta} \lambda+\sqrt{2} \theta \bar{\theta}\left(\left[\bar{z}_{i}, z_{i}\right]+i d\right)
$$

From the $\mathbf{S}$ superfield we can create the chiral and anti-chiral superfields which will appear in the action:

$$
\begin{aligned}
& \overline{\mathbf{\Upsilon}}=\frac{\mathcal{D} \mathbf{S}}{\sqrt{2}}=\psi_{3}+\bar{\theta}\left(\left[\bar{z}_{i}, z_{i}\right]-\left[\bar{z}_{3}, z_{3}\right]+i d\right)+\sqrt{2} \theta \bar{\theta}\left[\bar{z}_{3}, \psi_{3}\right] \\
& \mathbf{\Upsilon}=\frac{\overline{\mathcal{D}} \mathbf{S}}{\sqrt{2}}=\lambda-\theta\left(\left[\bar{z}_{i}, z_{i}\right]+\left[\bar{z}_{3}, z_{3}\right]+i d\right)-\sqrt{2} \theta \bar{\theta}\left[\bar{z}_{3}, \lambda\right]
\end{aligned}
$$

Note that $\bar{z}_{3}$ is a singlet under the two supersymmetries, and so is a superfield all by itself. Also note that the $\theta(\bar{\theta})$ components of (anti-)chiral superfields, and the $\bar{\theta} \theta$ components of a general superfield, transform under supersymmetry into a commutator; therefore the trace of such terms are supersymmetric invariants and are suitable for construction of the action.

In terms of these superfields, the action of the mother theory in Eq. 2.20) may be written as

$$
\begin{aligned}
S=\int & d \theta d \bar{\theta} \operatorname{Tr}\left(\frac{1}{2} \overline{\mathbf{\Upsilon}} \boldsymbol{\Upsilon}+\frac{1}{\sqrt{2}} \overline{\mathbf{Z}}_{i}\left[\mathbf{S}, \mathbf{Z}_{i}\right]-\frac{1}{2} \bar{\Xi} \boldsymbol{\Xi}\right) \\
& +\int d \theta \operatorname{Tr}\left(\boldsymbol{\Xi}\left[\mathbf{Z}_{1}, \mathbf{Z}_{2}\right]\right)-\int d \bar{\theta} \operatorname{Tr}\left(\bar{\Xi}\left[\overline{\mathbf{Z}}_{1}, \overline{\mathbf{Z}}_{2}\right]\right),
\end{aligned}
$$

The only difference between the above action and that of Eq. (2.20) is the addition of the auxiliary variables $d, G$ and $\bar{G}$, which only enter the action as

$$
S_{\mathrm{aux}}=\operatorname{Tr}\left[\frac{d^{2}}{2}+\bar{G} G\right],
$$

with the equations of motion $d=G=\bar{G}=0$, and have no effect on the dynamics of the theory. 
After performing the orbifold projection, the superfields take the form

$$
\begin{aligned}
& \mathbf{Z}_{i, \mathbf{n}}=z_{i, \mathbf{n}}+\sqrt{2} \theta \psi_{i, \mathbf{n}}-\sqrt{2} \theta \bar{\theta}\left(\bar{z}_{3, \mathbf{n}} z_{i, \mathbf{n}}-z_{i, \mathbf{n}} \bar{z}_{3, \mathbf{n}+\hat{\mathbf{e}}_{i}}\right), \\
& \overline{\mathbf{Z}}_{i, \mathbf{n}}=\bar{z}_{i, \mathbf{n}}+\sqrt{2} \bar{\theta} \epsilon_{i j} \xi_{j, \mathbf{n}}+\sqrt{2} \theta \bar{\theta}\left(\bar{z}_{3, \mathbf{n}+\hat{\mathbf{e}}_{i}} \bar{z}_{i, \mathbf{n}}-\bar{z}_{i, \mathbf{n}} \bar{z}_{3, \mathbf{n}}\right), \\
& \boldsymbol{\Xi}_{\mathbf{n}}=\xi_{3, \mathbf{n}}+\sqrt{2} \theta\left(G_{\mathbf{n}}-\sqrt{2} \epsilon_{i j} \bar{z}_{i, \mathbf{n}+\hat{\mathbf{e}}_{j}} \bar{z}_{j, \mathbf{n}}\right)-\sqrt{2} \theta \bar{\theta}\left(\bar{z}_{3, \mathbf{n}+\hat{\mathbf{e}}_{1}+\hat{\mathbf{e}}_{2}} \xi_{3, \mathbf{n}}-\bar{z}_{3, \mathbf{n}} \xi_{3, \mathbf{n}}\right), \\
& \bar{\Xi}_{\mathbf{n}}=\chi_{\mathbf{n}}-\sqrt{2} \bar{\theta}\left(\bar{G}_{\mathbf{n}}-\sqrt{2} \epsilon_{i j} z_{i, \mathbf{n}} z_{j, \mathbf{n}+\hat{\mathbf{e}}_{i}}\right)+\sqrt{2} \theta \bar{\theta}\left(\bar{z}_{3, \mathbf{n}} \chi_{\mathbf{n}}-\chi_{\mathbf{n}} \bar{z}_{\mathbf{n}+\hat{\mathbf{e}}_{1}+\hat{\mathbf{e}}_{2}}\right), \\
& \mathbf{S}_{\mathbf{n}}=z_{3, \mathbf{n}}+\sqrt{2} \theta \psi_{3, \mathbf{n}}+\sqrt{2} \bar{\theta} \lambda_{\mathbf{n}}+\sqrt{2} \theta \bar{\theta}\left(\bar{z}_{i, \mathbf{n}-\hat{\mathbf{e}}_{i}} z_{i, \mathbf{n}-\hat{\mathbf{e}}_{i}}-z_{i, \mathbf{n}} \bar{z}_{i, \mathbf{n}}+i d_{\mathbf{n}}\right), \\
& \mathbf{\Upsilon}_{\mathbf{n}}=\lambda_{\mathbf{n}}-\theta\left(\bar{z}_{i, \mathbf{n}-\hat{\mathbf{e}}_{i}} z_{i, \mathbf{n}-\hat{\mathbf{e}}_{i}}-z_{i, \mathbf{n}} \bar{z}_{i, \mathbf{n}}+\left[\bar{z}_{3, \mathbf{n}}, z_{3, \mathbf{n}}\right]+i d_{\mathbf{n}}\right)-\sqrt{2} \theta \bar{\theta}\left[\bar{z}_{3, \mathbf{n}}, \lambda_{\mathbf{n}}\right], \\
& \overline{\mathbf{\Upsilon}}_{\mathbf{n}}=\psi_{3, \mathbf{n}}+\bar{\theta}\left(\bar{z}_{i, \mathbf{n}-\hat{\mathbf{e}}_{i}} z_{i, \mathbf{n}-\hat{\mathbf{e}}_{i}}-z_{i, \mathbf{n}} \bar{z}_{i, \mathbf{n}}-\left[\bar{z}_{3, \mathbf{n}}, z_{3, \mathbf{n}}\right]+i d_{\mathbf{n}}\right)+\sqrt{2} \theta \bar{\theta}\left[\bar{z}_{3, \mathbf{n}}, \psi_{3, \mathbf{n}}\right] .
\end{aligned}
$$

The orbifold projection of the mother theory action in Eq. (3.11) may be written in terms of these lattice superfields as

$$
\begin{gathered}
S=\sum_{\mathbf{n}} \operatorname{Tr}\left[\int d \theta d \bar{\theta}\left(\frac{1}{2} \overline{\boldsymbol{\Upsilon}}_{\mathbf{n}} \mathbf{\Upsilon}_{\mathbf{n}}+\frac{1}{\sqrt{2}} \mathbf{S}_{\mathbf{n}}\left(\mathbf{Z}_{i, \mathbf{n}} \overline{\mathbf{Z}}_{i, \mathbf{n}}-\overline{\mathbf{Z}}_{i, \mathbf{n}-\hat{\mathbf{e}}_{i}} \mathbf{Z}_{i, \mathbf{n}-\hat{\mathbf{e}}_{i}}\right)-\frac{1}{2} \bar{\Xi}_{\mathbf{n}} \boldsymbol{\Xi}_{\mathbf{n}}\right)\right. \\
\left.+\int d \theta\left(\epsilon_{i j} \boldsymbol{\Xi}_{\mathbf{n}} \mathbf{Z}_{i, \mathbf{n}} \mathbf{Z}_{j, \mathbf{n}+\hat{\mathbf{e}}_{i}}\right)-\int d \bar{\theta}\left(\epsilon_{i j} \bar{\Xi}_{\mathbf{n}} \overline{\mathbf{Z}}_{i, \mathbf{n}+\hat{\mathbf{e}}_{j}} \overline{\mathbf{Z}}_{j, \mathbf{n}}\right)\right]
\end{gathered}
$$

\subsection{The $C_{2 v}$ and $U(1)^{4}$ symmetries of the $d=2$ lattice}

The action of the $C_{2 v}$ and $U(1)^{4}$ lattice symmetries are conveniently expressed in terms of superfields. The generators of $C_{2 v}$ are $C_{2}$, corresponding to rotations of the lattice by $\pi$, and $\sigma_{d}$, which reflects the lattice about the diagonal. Their effect on the various superfields of the theory are shown in Table 2. Note that this symmetry does not commute with supersymmetry, as the $C_{2}$ generator acts nontrivially on the Grassmann parameter $\theta$. Also shown in Table 2 are the charges of the fields under the $U(1)^{4}$ symmetry. We choose the four $U(1)$ charges to be $\mathbf{r}=\left\{r_{1}, r_{2}\right\}, q_{3}$ and $q_{4}$; the charges for the individual component variables were given in Table 1. Note that both $q_{3}$ and $q_{4}$ generate $R$-symmetries under which $\theta$ and $\bar{\theta}$ are charged. Furthermore, unlike in Minkowski space, one cannot find a linear combination of $q_{3}$ and $q_{4}$ which is not an $R$-symmetry, as $\theta$ and $\bar{\theta}$ are independent in Euclidean superspace. Also note that $q_{4}$ does not commute with the lattice symmetry transformation $C_{2}$.

\subsection{The continuum limit of the $d=2$ lattice}

The lattice action we have defined has a large classical moduli space, the space of possible values for our bosonic variables for which the ground state energy is zero. Following the procedure used in Refs. [1,2], we now expand our lattice action about the particular point in moduli space

$$
z_{1, \mathbf{n}}=z_{2, \mathbf{n}}=\frac{1}{\sqrt{2} a} \mathbf{1}_{k}
$$




\begin{tabular}{|c||c|c|c|c|c|c|c|c|c|c|c|}
\hline & $\theta$ & $\bar{\theta}$ & $\mathbf{Z}_{1, \mathbf{n}}$ & $\overline{\mathbf{Z}}_{1, \mathbf{n}}$ & $\mathbf{Z}_{2, \mathbf{n}}$ & $\overline{\mathbf{Z}}_{2, \mathbf{n}}$ & $\boldsymbol{\Xi}_{\mathbf{n}}$ & $\bar{\Xi}_{\mathbf{n}}$ & $\mathbf{S}_{\mathbf{n}}$ & $\mathbf{\Upsilon}_{\mathbf{n}}$ & $\bar{\Upsilon}_{\mathbf{n}}$ \\
\hline$C_{2}$ & $-\bar{\theta}$ & $-\theta$ & $\overline{\mathbf{Z}}_{1,-\mathbf{n}-\hat{\mathbf{e}}_{1}}$ & $\mathbf{Z}_{1,-\mathbf{n}-\hat{\mathbf{e}}_{1}}$ & $\overline{\mathbf{Z}}_{2,-\mathbf{n}-\hat{\mathbf{e}}_{2}}$ & $\mathbf{Z}_{2,-\mathbf{n}-\hat{\mathbf{e}}_{2}}$ & $\bar{\Xi}_{-\mathbf{n}}$ & $\boldsymbol{\Xi}_{-\mathbf{n}}$ & $\mathbf{S}_{-\mathbf{n}}$ & $-\overline{\mathbf{\Upsilon}}_{-\mathbf{n}}$ & $-\mathbf{\Upsilon}_{-\mathbf{n}}$ \\
$\sigma_{d}$ & $\theta$ & $\bar{\theta}$ & $\mathbf{Z}_{2, \tilde{\mathbf{n}}}$ & $\overline{\mathbf{Z}}_{2, \tilde{\mathbf{n}}}$ & $\mathbf{Z}_{1, \tilde{\mathbf{n}}}$ & $\overline{\mathbf{Z}}_{1, \tilde{\mathbf{n}}}$ & $-\boldsymbol{\Xi}_{\tilde{\mathbf{n}}}$ & $-\bar{\Xi}_{\tilde{\mathbf{n}}}$ & $\mathbf{S}_{\tilde{\mathbf{n}}}$ & $\mathbf{\Upsilon}_{\tilde{\mathbf{n}}}$ & $\overline{\boldsymbol{\Upsilon}}_{\tilde{\mathbf{n}}}$ \\
\hline $\mathbf{r}$ & 0 & 0 & $\{1,0\}$ & $\{-1,0\}$ & $\{0,1\}$ & $\{0,-1\}$ & $\{-1,-1\}$ & $\{1,1\}$ & $\{0,0\}$ & $\{0,0\}$ & $\{0,0\}$ \\
\hline$q_{3}$ & $\frac{1}{2}$ & $\frac{1}{2}$ & 0 & 0 & 0 & 0 & $\frac{1}{2}$ & $\frac{1}{2}$ & 1 & $\frac{1}{2}$ & $\frac{1}{2}$ \\
\hline$q_{4}$ & $-\frac{1}{2}$ & $\frac{1}{2}$ & 0 & 0 & 0 & 0 & $-\frac{1}{2}$ & $\frac{1}{2}$ & 0 & $-\frac{1}{2}$ & $\frac{1}{2}$ \\
\hline
\end{tabular}

Table 2: The action of the $C_{2}$ and $\sigma_{d}$ generators of the $C_{2 v}$ lattice symmetry on the superfields for the two-dimensional lattice of Fig. 1 , as well as the $U(1)^{4}$ charges taken to be $\mathbf{r}=\left\{r_{1}, r_{2}\right\}, q_{3}$ and $q_{4}$. The $\mathbf{r}$ charges correspond to the position of the superfield in the unit cell of the lattice. The coordinate used are $\mathbf{n}=\left\{n_{x}, n_{y}\right\}$ and $\tilde{\mathbf{n}}=\left\{n_{y}, n_{x}\right\}$. Neither the $C_{2}$ nor the $q_{4}$ charge commute with supersymmetry.

where $\mathbf{1}_{k}$ is the $k \times k$ unit matrix, and $\mathfrak{a}$ is interpreted as the lattice spacing. We obtain the continuum superfields by replacing the lattice coordinates $\mathbf{n}=\left\{n_{x}, n_{y}\right\}$ with continuous variables $\{x, y\}$, and shifting the fields

$$
\begin{array}{rlrl}
\boldsymbol{\Phi}_{1} & \equiv \mathbf{Z}_{1}-\frac{1}{\sqrt{2} \mathfrak{a}} \mathbf{1}_{k}, & \frac{\left(\phi_{1}+i v_{1}\right)}{\sqrt{2}} \equiv z_{1}-\frac{1}{\sqrt{2} \mathfrak{a}} \mathbf{1}_{k} \\
\boldsymbol{\Phi}_{2} \equiv \mathbf{Z}_{2}-\frac{1}{\sqrt{2} \mathfrak{a}} \mathbf{1}_{k}, & \frac{\left(\phi_{2}+i v_{2}\right)}{\sqrt{2}} \equiv z_{2}-\frac{1}{\sqrt{2} \mathfrak{a}} \mathbf{1}_{k},
\end{array}
$$

defining the superfields $\boldsymbol{\Phi}_{i}$ and the component fields $\phi_{i}, v_{i}$ (not to be confused with the gauge fields of the mother theory). Both $\phi_{i}$ and $v_{i}$ are Hermitean matrices. We then expand both the superfields in Eq. (3.13) and the lattice action Eq. (3.14) in powers of the lattice spacing $\mathfrak{a}^{1}$. Defining the covariant derivatives and field strength

$$
D_{1}=\partial_{1}+i v_{1}, \quad D_{2}=\partial_{2}+i v_{2}, \quad v_{12}=-i\left[D_{1}, D_{2}\right]
$$

\footnotetext{
${ }^{1}$ Although we do not show it here, the free spectrum of the lattice action does not have any fermion "doublers", so we are justified in keeping only smooth fields in the continuum limit, ignoring states near the edges of the Brillouin zone. How this works was shown explicitly for the $\mathcal{Q}=4$ theory of Ref. [2]
} 
we find the continuum superfields, up to terms of order $O(\mathfrak{a})$ :

$$
\begin{aligned}
\mathbf{\Phi}_{m} & =\frac{\left(\phi_{m}+i v_{m}\right)}{\sqrt{2}}+\sqrt{2} \theta \psi_{m}+\theta \bar{\theta}\left(D_{m} \bar{z}+\left[\phi_{m}, \bar{z}\right]\right)+O(\mathfrak{a}) \\
\overline{\mathbf{\Phi}}_{m} & =\frac{\left(\phi_{m}-i v_{m}\right)}{\sqrt{2}}+\sqrt{2} \bar{\theta} \epsilon_{m n} \xi_{n}+\theta \bar{\theta}\left(D_{m} \bar{z}-\left[\phi_{m}, \bar{z}\right]\right)+O(\mathfrak{a}) \\
\mathbf{S} & =z+\sqrt{2} \theta \psi_{3}+\sqrt{2} \bar{\theta} \lambda+\sqrt{2} \theta \bar{\theta}\left(-D_{1} \phi_{1}-D_{2} \phi_{2}+i d\right)+O(\mathfrak{a}) \\
\boldsymbol{\Xi} & =\xi_{3}+\theta\left(\sqrt{2} G+\left(D_{1} \phi_{2}-D_{2} \phi_{1}-i v_{12}-\left[\phi_{1}, \phi_{2}\right]\right)\right)-\sqrt{2} \theta \bar{\theta}\left[\bar{z}, \xi_{3}\right]+O(\mathfrak{a}) \\
\bar{\Xi} & =\chi+\bar{\theta}\left(-\sqrt{2} \bar{G}+\left(D_{1} \phi_{2}-D_{2} \phi_{1}+i v_{12}+\left[\phi_{1}, \phi_{2}\right]\right)\right)+\sqrt{2} \theta \bar{\theta}[\bar{z}, \chi]+O(\mathfrak{a}) \\
\mathbf{\Upsilon} & =\lambda+\theta\left(D_{1} \phi_{1}+D_{2} \phi_{2}-[\bar{z}, z]-i d\right)-\sqrt{2} \theta \bar{\theta}[\bar{z}, \lambda]+O(\mathfrak{a}) \\
\overline{\mathbf{\Upsilon}} & =\psi_{3}-\bar{\theta}\left(D_{1} \phi_{1}+D_{2} \phi_{2}+[\bar{z}, z]-i d\right)+\sqrt{2} \theta \bar{\theta}\left[\bar{z}, \psi_{3}\right]+O(\mathfrak{a})
\end{aligned}
$$

From the definition of the continuum superfields and the gauge transformation properties of the lattice variables, it is straightforward to determine how the continuum superfields vary under smooth gauge transformations. One finds that (up to $O(\mathfrak{a})$ corrections) all fields transform as $U(k)$ adjoints, except for the $\mathbf{\Phi}_{m}$ fields which transform inhomogeneously

$$
\boldsymbol{\Phi}_{m} \rightarrow U \boldsymbol{\Phi}_{m} U^{\dagger}+\frac{1}{\sqrt{2}} U \partial_{m} U^{\dagger}+O(\mathfrak{a}), \quad \overline{\mathbf{\Phi}}_{m} \rightarrow U \overline{\mathbf{\Phi}}_{m} U^{\dagger}-\frac{1}{\sqrt{2}} U \partial_{m} U^{\dagger}+O(\mathfrak{a}) .
$$

It follows that we can define the super-covariant derivatives

$$
\mathcal{D}_{m}=\partial_{m}+\sqrt{2} \boldsymbol{\Phi}_{m}, \quad \overline{\mathcal{D}}_{m}=-\partial_{m}+\sqrt{2} \overline{\mathbf{\Phi}}_{m},
$$

which transform as $\mathcal{D}_{m} \rightarrow U \mathcal{D}_{m} U^{\dagger}+O(\mathfrak{a})$, and similarly for $\overline{\mathcal{D}}_{m}$. From these one can construct chiral gauge field strength superfields $\mathcal{V}$ and $\overline{\mathcal{V}}$

$$
\begin{aligned}
\mathcal{V}_{m n}=- & i\left[\mathcal{D}_{m}, \mathcal{D}_{n}\right] \\
= & \left(v_{m n}-i\left(D_{m} \phi_{n}-D_{n} \phi_{m}\right)-i\left[\phi_{m}, \phi_{n}\right]\right) \\
& \quad-2 i \theta\left(D_{m} \psi_{n}-D_{n} \psi_{m}+\left[\phi_{m}, \psi_{n}\right]-\left[\phi_{n}, \psi_{m}\right]\right) \\
& +\sqrt{2} \theta \bar{\theta}\left[v_{m n}+i\left(D_{m} \phi_{n}-D_{n} \phi_{m}\right), \bar{z}\right] \\
\overline{\mathcal{V}}_{m n}=- & i\left[\overline{\mathcal{D}}_{m}, \overline{\mathcal{D}}_{n}\right] \\
= & \left(v_{m n}+i\left(D_{m} \phi_{n}-D_{n} \phi_{m}\right)-i\left[\phi_{m}, \phi_{n}\right]\right) \\
& \quad+2 i \bar{\theta}\left(D_{m} \epsilon_{n p} \xi_{p}-D_{n} \epsilon_{m p} \xi_{p}-\epsilon_{n p}\left[\phi_{m}, \xi_{p}\right]+\epsilon_{m p}\left[\phi_{n}, \psi_{p}\right]\right) \\
& \quad-\sqrt{2} \theta \bar{\theta}\left[v_{m n}+i\left(D_{m} \phi_{n}-D_{n} \phi_{m}\right), \bar{z}\right]
\end{aligned}
$$

as well as a "vector" gauge superfield strength $\mathcal{W}$ :

$$
\begin{aligned}
\mathcal{W}= & \sum_{m=1}^{2}\left[\mathcal{D}_{m}, \overline{\mathcal{D}}_{m}\right]=\sqrt{2} \partial_{m}\left(\boldsymbol{\Phi}_{m}+\overline{\mathbf{\Phi}}_{m}\right)+2\left[\boldsymbol{\Phi}_{m}, \overline{\mathbf{\Phi}}_{m}\right] \\
= & 2 D_{m} \phi_{m}+2 \theta\left(D_{m} \psi_{m}-\left[\phi_{m}, \psi_{m}\right]\right)+2 \bar{\theta} \epsilon_{m n}\left(D_{m} \xi_{n}+\left[\phi_{m}, \xi_{n}\right]\right) \\
& \quad+2 \sqrt{2} \theta \bar{\theta}\left(D_{m} D_{m} \bar{z}-\left[\phi_{m},\left[\phi_{m}, \bar{z}\right]\right]-\sqrt{2}\left\{\psi_{m}, \epsilon_{m n} \xi_{n}\right\}\right) .
\end{aligned}
$$


The action Eq. (3.14) may be compactly expressed in terms of these fields in a manifestly gauge and $\mathcal{Q}=2$ supersymmetric way as

$S=\frac{1}{2 g_{2}^{2}} \int d^{2} x \operatorname{Tr}\left[\int d \theta d \bar{\theta}\left(\overline{\mathbf{\Upsilon}} \boldsymbol{\Upsilon}+\frac{1}{\sqrt{2}} \mathbf{S} \mathcal{W}-\bar{\Xi} \boldsymbol{\Xi}\right)-i \int d \bar{\theta} \overline{\boldsymbol{\Xi}} \overline{\mathcal{V}}_{12}+i \int d \theta \boldsymbol{\Xi} \mathcal{V}_{12}\right]+O(\mathfrak{a})$

with $g_{2}^{2} \equiv g^{2} \mathfrak{a}^{2}$. After some algebra it is possible to show that the above action in the $\mathfrak{a} \rightarrow 0$ limit is identical to the action of the target theory (reproduced here from Eq. (1.1))

$S=\frac{1}{g_{2}^{2}} \int d^{2} x \operatorname{Tr}\left[\frac{1}{4} v_{m n} v_{m n}+\frac{1}{2}\left(D_{m} s_{a}\right)^{2}+\bar{\Psi}_{i} \gamma_{m} D_{m} \Psi_{i}+\bar{\Psi}_{i}\left[s_{0}, \Psi_{i}\right]+i \bar{\Psi}_{i} \gamma_{3} \boldsymbol{\tau}_{i j} \cdot\left[\boldsymbol{s}, \Psi_{j}\right]-\frac{1}{4}\left[s_{a}, s_{b}\right]^{2}\right]$

with the substitutions

$$
\begin{gathered}
\Psi_{1}=\left(\begin{array}{l}
\xi_{1} \\
\xi_{2}
\end{array}\right), \quad \Psi_{2}=\left(\begin{array}{c}
\lambda \\
-\xi_{3}
\end{array}\right), \quad \bar{\Psi}_{1}=\left(\begin{array}{ll}
-\chi \psi_{3}
\end{array}\right), \quad \bar{\Psi}_{2}=\left(\begin{array}{ll}
\psi_{1} & \psi_{2}
\end{array}\right) \\
\left(\begin{array}{c}
s_{0} \\
s_{1} \\
s_{2} \\
s_{3}
\end{array}\right)=\left(\begin{array}{c}
\phi_{1} \\
-(z+\bar{z}) / \sqrt{2} \\
i(z-\bar{z}) / \sqrt{2} \\
\phi_{2}
\end{array}\right)
\end{gathered}
$$

in the following $\gamma$-matrix basis:

$$
\gamma_{1}=-\sigma_{3}, \quad \gamma_{2}=-\sigma_{1}, \quad \gamma_{3}=\sigma_{2}
$$

It is not perversity that leads us to choose an off-diagonal matrix for the chirality matrix $\gamma_{3}-$ since chiral rotations are anomalous in the continuum theory, they mix fermion components from different locations on the lattice. As we chose a basis in Eq. (1.5) where each fermion component of the continuum corresponds to a particular lattice variable, the $\gamma_{3}$ matrix must necessarily be off-diagonal.

\subsection{Renormalization on the $d=2$ lattice}

The discussion of renormalization for this two dimensional lattice is similar to those given in Refs. $[1,2]$. We have shown that the tree level action has the desired continuum limit; we must now argue that there are no relevant or marginal operators generated radiatively which violate the Lorentz and $\mathcal{Q}=8$ supersymmetry of the target theory. An essential part of our argument relies on the fact that any such operators must be invariant under the exact $\mathcal{Q}=2$ supersymmetry, $U(k)$ gauge symmetry and the $C_{2 v}$ and $U(1)^{4}$ global symmetries respected by the lattice action.

Consider the addition to the action of chiral operators $\mathcal{O}$ and $\overline{\mathcal{O}}$, or a vector operator $\widehat{\mathcal{O}}$ :

$$
\delta S=\frac{1}{g_{2}^{2}} \int d^{2} x\left[\left(\int d \theta C \mathcal{O}+\int d \bar{\theta} \bar{C} \overline{\mathcal{O}}\right)+\int d \theta d \bar{\theta} \widehat{C} \widehat{\mathcal{O}}\right]
$$


We will ignore the anti-chiral operator $\overline{\mathcal{O}}$, which has the same power counting as the chiral operator $\mathcal{O}$ and is related by the $C_{2}$ lattice symmetry. For power counting purposes, $\frac{1}{g_{2}^{2}} \int d^{2} x$ has scaling dimension $-4, d \theta, d \bar{\theta}, \mathcal{D}$ and $\overline{\mathcal{D}}$ all scale with dimension $+\frac{1}{2}$. A contribution to any operator coefficient at $\ell$ loops is proportional to $g_{2}^{2 \ell}$. Since the coupling $g_{2}$ has mass dimension 1, the loop expansion is an expansion in powers of the dimensionless parameter $\left(g_{2}^{2} \mathfrak{a}^{2}\right)^{\ell}$. Consequently the operator coefficients have a loop expansion of the form

$$
\begin{aligned}
C & =\mathfrak{a}^{p-7 / 2} \sum_{\ell} c_{\ell}\left(g_{2}^{2} \mathfrak{a}^{2}\right)^{\ell}, \\
\widehat{C} & =\mathfrak{a}^{\widehat{p}-3} \sum_{\ell} \widehat{c}_{\ell}\left(g_{2}^{2} \mathfrak{a}^{2}\right)^{\ell},
\end{aligned}
$$

where the chiral and vector operators $\mathcal{O}$ and $\widehat{\mathcal{O}}$ are assumed to have mass dimension $p$ and $\widehat{p}$ respectively. The dimensionless expansion coefficients $c_{\ell}$ and $\widehat{c}_{\ell}$ depend at most logarithmically on the lattice spacing $\mathfrak{a}$. Considering $\ell \geq 1$, we see that the dangerous operators are the ones satisfying

$$
p-\frac{3}{2} \leq 0, \quad \widehat{p}-1 \leq 0
$$

The only possibilities for $\mathcal{O}$ on dimensional grounds are $\mathcal{O}=\operatorname{Tr} \boldsymbol{\Xi}$ or $\mathcal{O}=\operatorname{Tr} \Upsilon \boldsymbol{\Upsilon}$; however the former is excluded by its oddness under the $\sigma_{d}$ generator of the lattice $C_{2 v}$ symmetry (see Table 1), while the $\theta$ component of $\operatorname{Tr} \Upsilon$ is a total derivative and does not contribute to the action. As for the vector superfield operator operator, the only option is $\widehat{\mathcal{O}}=\operatorname{Tr} S$. This contributes a tadpole for the $U(1) d$-term, and so looks like a standard Fayet-Illiopoulos term, which in this case contributes only to a harmless cosmological constant. Thus the $\mathcal{Q}=8$ target theory is obtained without any fine tuning of parameters, since no marginal or relevant operators spoil its emergence in the $\mathfrak{a} \rightarrow 0$ limit.

\subsection{Fixing the moduli}

The last loose end to tie up is the fixing of moduli (bosonic zeromodes). The analysis is identical to that in $\S 6$ of Ref. [2], and so we only recapitulate the results here. The first point is that fixing the "vacuum expectation values" of the $z_{1, \mathbf{n}}$ and $z_{2, \mathbf{n}}$ link fields as in Eq. (3.15) requires the addition of a supersymmetry breaking term to the action which lifts the degeneracy of the moduli. In terms of the continuum variables it takes the form of a small mass term $\mu$ for the $s_{a}$ boson quartet, which vanishes in the large volume limit. In terms of the lattice variables, such a term can take the form of the last line in Eq. (1.1):

$$
\frac{a^{2} \mu^{2}}{2}\left[\left(z_{1, \mathbf{n}} \bar{z}_{1, \mathbf{n}}-\frac{1}{2 a^{2}}\right)^{2}+\left(z_{2, \mathbf{n}} \bar{z}_{2, \mathbf{n}}-\frac{1}{2 a^{2}}\right)^{2}+2 \frac{\bar{z}_{3, \mathbf{n}} z_{3, \mathbf{n}}}{a^{2}}\right]
$$

The parameter $\mu$ can be taken to be $\lesssim 1 / L=1 / N \mathfrak{a}$. The mass terms in Eq. (3.31) serve to fix the scalar zeromodes, just as an external magnetic field whose strength scales to zero in the large volume limit can be used to study systems with spontaneous magnetization. There 
is the separate issue of the infrared divergences of nonzero momentum modes of the moduli, which one might expect to be severe in two dimensions, spoiling our expansion about $\left\langle z_{1, \mathbf{n}}\right\rangle=$ $\left\langle z_{2, \mathbf{n}}\right\rangle=\frac{1}{2 \sqrt{\mathfrak{a}}}$; however, as shown in Ref. [2] this is in fact not a problem with our method, provided the continuum and large volume limits are taken so that $\mathfrak{a}^{2} g_{2}^{2} \ln N=\mathfrak{a}^{4} g^{2} \ln N \rightarrow 0$.

\section{The three dimensional lattice}

\subsection{The $d=3$ lattice action and its symmetries}

The three dimensional lattice is derived from the $\mathcal{Q}=8$ mother theory by performing a

$Z_{N}^{3}$ orbifold projection, where the $Z_{N}^{3}$ symmetry is defined by the charges $r_{1,2,3}$ in Table 1. This lattice will describe the $\mathcal{N}=4$ supersymmetry in three dimensions in the continuum, whose action is given Eq. (1.7). The lattice we obtain takes the form shown in Fig. 2, with $\lambda$ residing at the sites; $\left\{z_{a}, \bar{z}_{a}, \psi_{a}\right\}$ on the $\hat{\mathbf{z}}_{a}$-links for $a=1,2,3$; the $\xi_{a}$ on the diagonal face links; and $\chi$ on the superdiagonal link. This lattice possesses a $C_{3 v} \cong S_{3}$ point group symmetry, consisting of $2 \pi / 3$ rotations around the $\chi$ link, as well as reflections about the three planes containing both $\chi$ and $\psi_{i}$ links. The lattice action is also invariant under a single supersymmetry transformation, obtained by setting $\kappa$ in the supersymmetry transformations of the mother theory Eq. (2.7) to

$$
\kappa=\left(\begin{array}{ll}
\eta & 0 \\
0 & 0 \\
0 & 0 \\
0 & 0
\end{array}\right)
$$

Following the procedure followed for the two dimensional lattice, we introduce a Grassmann coordinate $\theta$ and define the supersymmetry transformation to be

$$
\delta=i \eta Q, \quad Q=\frac{\partial}{\partial \theta}
$$

which acts on the superfields

$$
\begin{aligned}
\boldsymbol{\Lambda}_{\mathbf{n}} & =\lambda_{\mathbf{n}}-\theta\left(\bar{z}_{a, \mathbf{n}-\hat{\mathbf{e}}_{a}} z_{a, \mathbf{n}-\hat{\mathbf{e}}_{a}}-z_{a, \mathbf{n}} \bar{z}_{a, \mathbf{n}}+i d_{\mathbf{n}}\right), \\
\mathbf{Z}_{a, \mathbf{n}} & =z_{a, \mathbf{n}}+\sqrt{2} \theta \psi_{a, \mathbf{n}}, \\
\boldsymbol{\Xi}_{a, \mathbf{n}} & =\xi_{a, \mathbf{n}}-2 \theta \epsilon_{a b c} \bar{z}_{b, \mathbf{n}+\hat{\mathbf{e}}_{c}} \bar{z}_{c, \mathbf{n}} .
\end{aligned}
$$

In the above equation repeated indices are summed over $1,2,3$, and $\hat{\mathbf{e}}_{a}$ is a unit lattice vector in the positive $z_{a}$ direction. In terms of individual components, the supersymmetry 
transformations are given by

$$
\begin{aligned}
& \delta z_{a, \mathbf{n}}=i \sqrt{2} \eta \psi_{a, \mathbf{n}} \\
& \delta \bar{z}_{a, \mathbf{n}}=0 \\
& \delta \psi_{a, \mathbf{n}}=0 \\
& \delta \xi_{a, \mathbf{n}}=-2 i \eta \epsilon_{a b c}\left(\bar{z}_{b, \mathbf{n}+\hat{\mathbf{e}}_{c}} \bar{z}_{c, \mathbf{n}}\right) \\
& \delta \lambda_{\mathbf{n}}=-i \eta\left(\bar{z}_{a, \mathbf{n}-\hat{\mathbf{e}}_{a}} z_{a, \mathbf{n}-\hat{\mathbf{e}}_{a}}-z_{a, \mathbf{n}} \bar{z}_{a, \mathbf{n}}+i d_{\mathbf{n}}\right) \\
& \delta \chi_{\mathbf{n}}=0 \\
& \delta d_{\mathbf{n}}=-\sqrt{2} \eta\left(\bar{z}_{a, \mathbf{n}-\hat{\mathbf{e}}_{a}} \psi_{a, \mathbf{n}-\hat{\mathbf{e}}_{a}}-\psi_{a, \mathbf{n}} \bar{z}_{a, \mathbf{n}}\right) .
\end{aligned}
$$

Note that $\bar{z}_{a}, \psi_{a}$ and $\chi$ are all supersymmetric singlets, as is the $\theta$ component of any superfield.

The lattice action we obtain may be written in manifestly $\mathcal{Q}=1$ supersymmetric form as

$$
\begin{aligned}
S=\frac{1}{g^{2}} \sum_{\mathbf{n}} \operatorname{Tr} & \left(\int d \theta\left[-\frac{1}{2} \boldsymbol{\Lambda}_{\mathbf{n}} \partial_{\theta} \boldsymbol{\Lambda}_{\mathbf{n}}-\boldsymbol{\Lambda}_{\mathbf{n}}\left(\bar{z}_{a, \mathbf{n}-\hat{\mathbf{e}}_{a}} \mathbf{Z}_{a, \mathbf{n}-\hat{\mathbf{e}}_{a}}-\mathbf{Z}_{a, \mathbf{n}} \bar{z}_{a, \mathbf{n}}\right)+\epsilon_{a b c} \boldsymbol{\Xi}_{a, \mathbf{n}} \mathbf{Z}_{b, \mathbf{n}} \mathbf{Z}_{c, \mathbf{n}+\hat{\mathbf{e}}_{b}}\right]\right. \\
& \left.-\sqrt{2} \chi_{\mathbf{n}}\left(\bar{z}_{a, \mathbf{n}+\boldsymbol{\omega}_{a}} \boldsymbol{\Xi}_{a, \mathbf{n}}-\boldsymbol{\Xi}_{a, \mathbf{n}+\hat{\mathbf{e}}_{a}} \bar{z}_{a, \mathbf{n}}\right)\right)
\end{aligned}
$$

with

$$
\boldsymbol{\omega}_{a} \equiv \sum_{b \neq a} \hat{\mathbf{e}}_{b}=\{1,1,1\}-\hat{\mathbf{e}}_{a} .
$$

The last term in the action is not integrated over $\theta$, even though it contains the nontrivial superfield $\boldsymbol{\Xi}$. However, due to the $\epsilon_{a b c}$ tensor in the $\theta$ component of $\boldsymbol{\Xi}$, one can see that the $\theta$ component of the $\operatorname{Tr} \chi[\bar{z}, \boldsymbol{\Xi}]$ operator in the action above identically vanishes, so that $\operatorname{Tr} \chi_{\mathbf{n}}\left(\bar{z}_{a, \mathbf{n}+\boldsymbol{\omega}_{a}} \boldsymbol{\Xi}_{a, \mathbf{n}}-\boldsymbol{\Xi}_{a, \mathbf{n}+\hat{\mathbf{e}}_{a}} \bar{z}_{a, \mathbf{n}}\right)$ is $\theta$-independent and hence supersymmetric.

The transformations of the superfields under the global symmetries of the theory are given in Table 3. In this table the $S_{3}$ generators $R$ and $\Sigma$ are given by

$$
R=\left(\begin{array}{lll}
0 & 1 & 0 \\
0 & 0 & 1 \\
1 & 0 & 0
\end{array}\right) \quad \Sigma=\left(\begin{array}{lll}
0 & 1 & 0 \\
1 & 0 & 0 \\
0 & 0 & 1
\end{array}\right) .
$$

\subsection{The continuum limit of the $d=3$ lattice}

As we did for the two dimensional lattice, we now reexpress the three dimensional lattice theory in terms of continuum superfields which facilitates the analysis of its properties under renormalization. We define the shifted link fields

$$
\frac{\left(\phi_{a}+i v_{a}\right)}{\sqrt{2}} \equiv z_{a}-\frac{1}{\sqrt{2} \mathfrak{a}} \mathbf{1}_{k}, \quad \mathbf{\Phi}_{a} \equiv \mathbf{Z}_{a}-\frac{1}{\sqrt{2} \mathfrak{a}}=\frac{\left(\phi_{a}+i v_{a}\right)}{\sqrt{2}}+\sqrt{2} \theta \psi_{a},
$$




\begin{tabular}{|c||c|c|c|c|c|c|}
\hline & $\theta$ & $\mathbf{Z}_{a, \mathbf{n}}$ & $\bar{z}_{a, \mathbf{n}}$ & $\boldsymbol{\Xi}_{a, \mathbf{n}}$ & $\Lambda_{\mathbf{n}}$ & $\chi_{\mathbf{n}}$ \\
\hline \hline$C_{3}$ & $\theta$ & $R_{a b} \mathbf{Z}_{b, R \mathbf{n}}$ & $R_{a b} \bar{z}_{b, R \mathbf{n}}$ & $R_{a b} \boldsymbol{\Xi}_{b, R \mathbf{n}}$ & $\Lambda_{\mathbf{n}}$ & $\chi_{\mathbf{n}}$ \\
\hline$\sigma_{3}$ & $\theta$ & $\Sigma_{a b} \mathbf{Z}_{b, \Sigma \mathbf{n}}$ & $\Sigma_{a b} \bar{z}_{b, \Sigma \mathbf{n}}$ & $-\Sigma_{a b} \boldsymbol{\Xi}_{b, \Sigma \mathbf{n}}$ & $\Lambda_{\mathbf{n}}$ & $\chi_{\mathbf{n}}$ \\
\hline $\mathbf{r}$ & $\mathbf{0}$ & $\hat{\mathbf{e}}_{a}$ & $-\hat{\mathbf{e}}_{a}$ & $\hat{\mathbf{e}}_{a}-\sum_{b} \hat{\mathbf{e}}_{b}$ & $\mathbf{0}$ & $\sum_{b} \hat{\mathbf{e}}_{b}$ \\
\hline$q_{4}$ & $-\frac{1}{2}$ & 0 & 0 & $-\frac{1}{2}$ & $-\frac{1}{2}$ & $\frac{1}{2}$ \\
\hline
\end{tabular}

Table 3: The transformation properties of fields on the three dimensional lattice under the global $S_{3} \times U(1)^{4}$ symmetry. $C_{3}$ and $\sigma_{3}$ generate the $S_{3}$ group, with the former consisting of $2 \pi / 3$ rotations about the vector $\{1,1,1\}$, and $\sigma_{3}$ corresponding to reflections about the plane containing the $\{0,0,1\}$ and $\{1,1,1\}$ vectors. The matrices $R$ and $\Sigma$ are given in the text. The global $U(1)^{4}$ symmetry is taken to be generated by the three $\mathbf{r}$ charges, and $q_{4}$, the latter being an $R$-charge on the lattice.

where the subscript $a$ runs over $1,2,3$. The ordinary gauge covariant derivatives and field strength are defined as

$$
D_{m}=\partial_{m}+i v_{m}, \quad v_{m n}=-i\left[D_{m}, D_{n}\right] .
$$

where the spacetime indices $m, n=1,2,3$. The continuum limit of the lattice fields involves keeping only the smooth configurations, as explicit computation shows that the perturbative propagators exhibit no poles near the edges of the Brillouin zone. We can write the continuum superfields (up to $O(\mathfrak{a})$ corrections) as

$$
\begin{aligned}
\boldsymbol{\Phi}_{a} & =\frac{\phi_{a}+i v_{a}}{\sqrt{2}}+\sqrt{2} \theta \psi_{a}, \\
\boldsymbol{\Xi}_{a} & =\xi_{a}+\frac{1}{2} \theta \epsilon_{a b c}\left(D_{b} \phi_{c}-D_{c} \phi_{b}-\left[\phi_{b}, \phi_{c}\right]-i v_{b c}\right)+O(\mathfrak{a}), \\
\boldsymbol{\Lambda} & =\lambda+\theta\left(D_{a} \phi_{a}-i d\right)+O(\mathfrak{a}),
\end{aligned}
$$

where each field transforms as an adjoint under the $U(k)$ gauge symmetry, and is a function of the three coordinates in our three dimensional Euclidean spacetime. The lattice action is again most easily written by introducing super-covariant derivatives, which in the present case are

$$
\mathcal{D}_{m} \equiv \partial_{m}+\sqrt{2} \Phi_{m}, \quad \overline{\mathcal{D}}_{m} \equiv-\partial_{m}+\left(\phi_{m}-i v_{m}\right)=-D_{m}+\phi_{m} .
$$

From these one can construct the field strengths

$$
\begin{aligned}
\mathcal{V}_{m n} & =-i\left[\mathcal{D}_{m}, \mathcal{D}_{n}\right] \\
& =-i\left(D_{m} \phi_{n}-D_{n} \phi_{n}+\left[\phi_{m}, \phi_{n}\right]+i v_{m n}\right)-2 i \theta\left(D_{m} \psi_{n}-D_{n} \psi_{m}+\left[\psi_{m}, \phi_{n}\right]-\left[\psi_{n}, \phi_{m}\right]\right), \\
\mathcal{W} & =\left[\mathcal{D}_{m}, \overline{\mathcal{D}}_{m}\right]=2 D_{m} \phi_{m}+2 \theta\left(D_{m} \psi_{m}+\left[\psi_{m}, \phi_{m}\right]\right),
\end{aligned}
$$


where $m$ is summed over $1,2,3$ in the expression for $\mathcal{W}$. The lattice action Eq. (4.5) may then be rewritten in terms of continuum fields, with $g_{3}^{2} \equiv \mathfrak{a}^{3} g^{2}$, as

$$
S=\frac{1}{g_{3}^{2}} \int d^{3} x \operatorname{Tr}\left(-\chi\left[\overline{\mathcal{D}}_{a}, \boldsymbol{\Xi}_{a}\right]+\int d \theta\left[-\frac{1}{2} \Lambda \partial_{\theta} \Lambda+\frac{1}{2} \Lambda \mathcal{W}+\frac{i}{4} \epsilon_{a b c} \boldsymbol{\Xi}_{a} \mathcal{V}_{b c}\right]\right)+O(\mathfrak{a})
$$

In terms of components, after eliminating the auxiliary field $d$, this yields the desired target theory

$$
S=\frac{1}{g_{3}^{2}} \int d^{3} x \operatorname{Tr}\left[\frac{1}{4} v_{m n} v_{m n}+\frac{1}{2}\left(D_{m} \phi_{a}\right)^{2}+\bar{\Psi}_{i} \sigma_{m} D_{m} \Psi_{i}-\bar{\Psi}_{i} \tau_{i j}^{a} \cdot\left[\phi_{a}, \Psi_{j}\right]-\frac{1}{4}\left[\phi_{a}, \phi_{b}\right]^{2}\right] .
$$

where the fermions fields are given by

$$
\begin{aligned}
& \Psi_{1}=\frac{1}{\sqrt{2}}\left(\begin{array}{c}
\psi_{3}-i \chi \\
\psi_{1}+i \psi_{2}
\end{array}\right), \quad \bar{\Psi}_{1}=\frac{i}{\sqrt{2}}\left(\begin{array}{c}
\xi_{3}+i \lambda \\
\xi_{1}-i \xi_{2}
\end{array}\right)^{T} \\
& \Psi_{2}=\frac{1}{\sqrt{2}}\left(\begin{array}{c}
-\psi_{1}+i \psi_{2} \\
\psi_{3}+i \chi
\end{array}\right), \quad \bar{\Psi}_{2}=\frac{i}{\sqrt{2}}\left(\begin{array}{c}
-\xi_{1}-i \xi_{2} \\
\xi_{3}-i \lambda
\end{array}\right)^{T}
\end{aligned}
$$

in a basis where the three gamma matrices are just the Pauli matrices,

$$
\gamma_{m}=\sigma_{m}
$$

\subsection{Renormalization on the $d=3$ lattice}

To find out whether operators might be radiatively induced which could spoil the continuum limit, we follow the same procedure as in $\S 3.4$. Counterterms in the action will take the generic form ${ }^{2}$

$$
\delta S=\frac{1}{g_{3}^{2}} \int d^{3} x\left[\int d \theta C \mathcal{O}\right]
$$

where $\mathcal{O}$ is an operator of dimension $p$. The coupling $g_{3}^{2}$ scales like mass, while $d^{3} x$ and $d \theta$ have mass dimension $(-3)$ and $1 / 2$ respectively. Then on dimensional grounds, contributions to $C$ in a loop expansion are of the form

$$
C=\mathfrak{a}^{p-7 / 2} \sum_{\ell} c_{\ell}\left(g_{3}^{2} \mathfrak{a}\right)^{\ell}
$$

where $\ell$ counts the number of loops and $c_{\ell}$ is dimensionless, depending at most logarithmically on $\mathfrak{a}$. If $\mathcal{O}$ is an operator which violates the symmetries of the target theory then its coefficient

\footnotetext{
${ }^{2}$ One might wonder if there could be operators induced which do not have to be integrated over $\theta$, such as the first one in Eq. (4.13). However, it is not hard to show that there are no such operators that satisfy our criteria for "dangerous operators", due to the combined strictures of gauge invariance and the $S_{3}$ symmetry.
} 
should vanish in the $\mathfrak{a} \rightarrow 0$ limit, or the continuum limit will be spoiled. Since radiative corrections begin at $\ell=1$, it follows that we need to check whether the theory allows operators with $p \leq \frac{5}{2}$ which respect the exact symmetries of the lattice.

The operator $\mathcal{O}$ must be Grassmann, and hence its dimension must be half integer. It must have $U(1)$ charge $q_{4}=-\frac{1}{2}$, and be invariant under the gauge and $S_{3}$ symmetries. Denoting generic bosonic superfields as $B$ and fermion superfields as $F$, we find that there is no operator of dimension $p \leq \frac{1}{2}$; at dimensions $p=\frac{3}{2} \mathcal{O}$ must take the form $\partial_{\theta} B$ or $F$. The former is a total derivative, but the latter is a possibility. However when the symmetries are taken into account, the only possible operator one could add is $\mathcal{O}=\operatorname{Tr} \Lambda$. This is a FayetIliopoulos term, and as discussed previously, it only contributes to a cosmological constant, and does not affect the excitations of theory.

At dimension $p=\frac{5}{2}$ the choices for $\mathcal{O}$ are $B \partial_{\theta} B^{\prime}$ and $F B$. The former can be ruled out as there is no operator of that form with $q_{4}=-\frac{1}{2}$. However there are two similar operators of the form $F B$ which are invariant under all the symmetries, namely

$$
\mathcal{O}_{1}=\frac{1}{\sqrt{2}} \sum_{a} \operatorname{Tr} \Lambda\left(\mathbf{\Phi}_{a}+\bar{\phi}_{a}\right), \quad \text { and } \quad \mathcal{O}_{2}=\frac{1}{\sqrt{2}} \sum_{a} \operatorname{Tr} \Lambda \operatorname{Tr}\left(\mathbf{\Phi}_{a}+\bar{\phi}_{a}\right)
$$

Note that $\Phi_{a}$ and $\bar{\phi}_{a}$ must appear in this combination for the inhomogeneous shift under gauge transformations to cancel. In component form, these operators are equivalent to $\sum_{a} \operatorname{Tr}\left(-\lambda \psi_{a}+\left(D_{b} \phi_{b}-i d\right) \phi_{a}\right)$ and its double trace analogue. On the elimination of $d$, such operators would induce the scalar mass term $\operatorname{Tr}\left(\sum_{a} \phi_{a}\right)^{2}$. The coefficients of these operators can have at most a logarithmic divergence at one loop, and vanishing contributions at higher loops. Therefore, while we cannot rule out that these operators could be radiatively induced, in principle the critical couplings can be determined from a one-loop calculation on the lattice.

The discussion about how to fix the moduli of the three dimensional theory is similar to that given in $\S 3.5$ for the two dimensional case, and we will not repeat it here.

In conclusion, the three dimensional lattice allows two supersymmetric counterterms which could be radiatively induced at one loop, and which would spoil the continuum limit. The coefficient of this operator only runs logarithmically with scale, and it must be tuned to the critical point, either numerically, or theoretically by performing a one loop computation on the lattice.

\section{Discussion}

We have explicitly constructed a nonperturbative regulator for quantum field theories with eight supercharges in two and three Euclidean dimensions. Although there may be challenges to overcome, such as a potential sign problem with the fermion determinant ${ }^{3}$ (as there is in the two dimensional theory with four supercharges [9]), we hope that eventually these lattice may be of use for numerical simulations. While there is a substantial literature on

\footnotetext{
${ }^{3}$ J. Giedt, private communication
} 
these theories already, most previous theoretical investigations have centered on trying to understand the structure of their moduli spaces. Presumably numerical simulations would begin by establishing that the supersymmetric Ward identities are satisfied, and proceed with a study of the particle spectrum, about which little is known.

We are optimistic that these lattices could prove useful for theoretical investigations as well, such as furthering our understanding of mirror symmetry in three dimensions [15], or leading to the construction of a nonperturbative Nicolai map [16] in a fully regulated supersymmetric gauge theory.

A final paper in this series in preparation will address the construction of spacetime lattices for theories with sixteen supercharges.

\section{Acknowledgments}

D.B.K. and M.U. were supported in part by DOE grant DE-FGO3-00ER41132, E.K by DOE grant DE-FG03-96ER40956, and A.G.C. by DOE grant DE-FG03-91ER-40676.

\section{References}

[1] D. B. Kaplan, E. Katz, and M. Unsal, Supersymmetry on a spatial lattice, JHEP 05 (2003) 037, hep-lat/0206019.

[2] A. G. Cohen, D. B. Kaplan, E. Katz, and M. Unsal, Supersymmetry on a euclidean spacetime lattice I: A target theory with four supercharges, http://arXiv.org/abs/hep-lat/0302017.

[3] M. R. Douglas and G. W. Moore, D-branes, quivers, and ale instantons, http://arXiv.org/abs/hep-th/9603167.

[4] N. Arkani-Hamed, A. G. Cohen, and H. Georgi, (De)constructing dimensions, Phys. Rev. Lett. 86 (2001) 4757-4761, http://arXiv.org/abs/hep-th/0104005.

[5] N. Arkani-Hamed, A. G. Cohen, D. B. Kaplan, A. Karch, and L. Motl, Deconstructing (2,0) and little string theories, http://arXiv.org/abs/hep-th/0110146.

[6] D. B. Kaplan, A method for simulating chiral fermions on the lattice, Phys. Lett. B288 (1992) 342-347, http://arXiv.org/abs/hep-lat/9206013.

[7] R. Narayanan and H. Neuberger, A construction of lattice chiral gauge theories, Nucl. Phys. B443 (1995) 305-385, http://arXiv.org/abs/hep-th/9411108.

[8] H. Neuberger, Exactly massless quarks on the lattice, Phys. Lett. B417 (1998) 141-144, http://arXiv.org/abs/hep-lat/9707022.

[9] J. Giedt, Non-positive fermion determinants in lattice supersymmetry, hep-lat/0304006.

[10] D.-E. Diaconescu and N. Seiberg, The coulomb branch of $(4,4)$ supersymmetric field theories in two dimensions, JHEP 07 (1997) 001, hep-th/9707158.

[11] E. Witten, On the conformal field theory of the higgs branch, JHEP 07 (1997) 003, hep-th/9707093. 
[12] N. Seiberg, IR dynamics on branes and space-time geometry, Phys. Lett. B384 (1996) 81-85, hep-th/9606017.

[13] N. Seiberg and E. Witten, Gauge dynamics and compactification to three dimensions, hep-th/9607163.

[14] G. Chalmers and A. Hanany, Three dimensional gauge theories and monopoles, Nucl. Phys. B489 (1997) 223-244, hep-th/9608105.

[15] A. Kapustin and M. J. Strassler, On mirror symmetry in three dimensional abelian gauge theories, JHEP 04 (1999) 021, hep-th/9902033.

[16] H. Nicolai, Supersymmetry and functional integration measures, Nucl. Phys. B176 (1980) 419-428. 